\title{
Deep-inelastic electron-deuteron scattering with spectator nucleon tagging at the future Electron Ion Collider: Extracting free nucleon structure
}

\author{
Alexander Jentsch $\odot,{ }^{1, *}$ Zhoudunming Tu, ${ }^{1,2, \dagger}$ and Christian Weiss ${ }^{3, \dagger}$ \\ ${ }^{1}$ Department of Physics, Brookhaven National Laboratory, Upton, New York 11973, USA \\ ${ }^{2}$ Center for Frontiers in Nuclear Science, Stony Brook, New York 11794, USA \\ ${ }^{3}$ Theory Center, Jefferson Lab, Newport News, Virginia 23606, USA
}

(Received 25 August 2021; revised 10 November 2021; accepted 30 November 2021; published 27 December 2021)

\begin{abstract}
Background: Deep-inelastic scattering (DIS) on the deuteron with spectator nucleon tagging represents a unique method for extracting the free neutron structure functions and exploring the nuclear modifications of bound protons and neutrons. The detection of the spectator (with typical momentum $\lesssim 100 \mathrm{MeV} / c$ in the deuteron rest frame) controls the nuclear configuration during the DIS process and enables a differential analysis of nuclear effects. At the future Electron Ion Collider (EIC) such measurements will be performed using far-forward detectors.

Purpose: Simulate deuteron DIS with proton or neutron tagging with the baseline EIC far-forward detector design. Quantify detector acceptance and resolution effects. Study feasibility of free nucleon structure extraction using pole extrapolation in the spectator momentum.

Methods: DIS events with proton and neutron spectators are generated using the BeAGLE Monte Carlo generator. The spectator nucleon momentum is reconstructed including effects of detector acceptance and resolution. Pole extrapolation is performed under realistic conditions. The free nucleon structure extraction is validated by comparing with the input model.

Results: Proton and neutron spectator detection is possible over the full transverse momentum range $0<p_{T}<$ $100 \mathrm{MeV} / c$ needed for pole extrapolation. Resolution effects on the distributions before corrections are $\approx 10 \%$ for proton and $\approx 30 \%$ for neutron spectators. The overall accuracy of nucleon structure extraction is expected to be at the few-percent level.

Conclusions: Free neutron structure extraction through proton tagging and pole extrapolation is feasible with the baseline EIC far-forward detector design. The corresponding extraction of free proton structure through neutron tagging provides a reference point for future studies of nuclear modifications.
\end{abstract}

DOI: 10.1103/PhysRevC.104.065205

\section{INTRODUCTION}

Deep-inelastic lepton scattering (DIS) represents a principal tool for exploring the short-range structure of hadrons and nuclei and studying the expressions of quantum chromodynamics in the perturbative and nonperturbative regimes. DIS measurements are performed on the proton, light ions $(2 \leqslant A \lesssim 12)$, and heavy ions, with complementary physics purposes. DIS measurements on light ions pursue several specific objectives. One objective is to extract the DIS observables of the neutron, to enable the flavor separation of the

\footnotetext{
*ajentsch@bnl.gov

tzhoudunming@bnl.gov

‡weiss@jlab.org
}

Published by the American Physical Society under the terms of the Creative Commons Attribution 4.0 International license. Further distribution of this work must maintain attribution to the author(s) and the published article's title, journal citation, and DOI. Funded by $S C O A P^{3}$. nucleon's partonic structure expressed in the parton distribution functions (PDFs) [1-3], generalized parton distributions (GPDs) [4-7], and transverse momentum dependent structures (TMDs) [8-10]. A second objective is to study the nuclear modifications of partonic structure (EMC effect at $x>0.3$, antishadowing at $x \approx 0.1$ ), in order to explain their dynamical origin and connection with conventional nuclear interactions [11-14]. A third objective is to measure coherent and diffractive scattering on light nuclei, in order to characterize the quark/gluon structure of the nucleus in novel ways [15], and to observe the onset of the coherent phenomena expected in heavy nuclei at small $x$ (shadowing, diffraction) $[16,17]$.

The future Electron Ion Collider (EIC) will enable a comprehensive program of DIS measurements on light ions. The accelerator design provides light ion beams of several species, including the deuteron $d \equiv{ }^{2} \mathrm{H},{ }^{3} \mathrm{He}$, and ${ }^{4} \mathrm{He}$. It supports electron-ion collisions in a broad range of center-of-mass energies, $\approx 20-100 \mathrm{GeV} /$ nucleon for electron-deuteron, at luminosities $\approx 10^{33}-10^{34} \mathrm{~cm}^{-2} \mathrm{~s}^{-1}$; for more details on the capabilities for protons, light ion, and heavy ion beams see Ref. [18]. Ion polarization will be available for the ${ }^{3} \mathrm{He}$ beams, 
and possibly also for the $d$. DIS-type measurements on light ions will be performed with inclusive, semi-inclusive, and exclusive final states. The EIC central detector will provide excellent coverage for the scattered electron and the current fragmentation region of the DIS final states. In addition, a suite of optimized far-forward detectors (pseudorapidity $\eta>$ 4.5) will enable detection of the nuclear breakup state and/or the identification of coherent nuclear events. A description of the proposed reference detector and detailed discussion of the requirements can be found in the recently completed EIC Yellow Report [19].

The main challenge in the interpretation of DIS measurements on light nuclei lies in the treatment of nuclear binding effects. The nucleus participates in the DIS process in a variety of nuclear configurations characterized by the nucleon momenta, spins, interactions, and non-nucleonic degrees of freedom; in a quantum-mechanical superposition described by the nuclear wave function. The nuclear binding effects one needs to account for generally depend on the nuclear configuration. In neutron structure extraction one needs to correct for dilution from scattering on the protons and eliminate effects of nucleon motion and interactions. In studies of the EMC effect one wants to connect the observed modifications of partonic structure with a particular range of nucleon momenta or distances and the interactions between them (e.g., a possible connection with short-range nucleon-nucleon correlations [20]). With inclusive nuclear DIS measurements, where no detection of the nuclear breakup state is performed, one has no information on the nuclear configurations during the DIS process and must model the nuclear binding effects in all possible configurations and sum over them, resulting in large theoretical uncertainties. This problem can be overcome with tagged measurements, where one detects part or all of the nuclear breakup state, so that one can use the breakup observables to infer the nuclear configuration during the DIS process. In this way one can effectively control the nuclear configuration during the DIS process and treat the nuclear effects in defined configurations. In neutron structure extraction, one can select configurations where the neutron is effectively free. In studies of the EMC effect, one can select configurations with definite nucleon momenta/distances and control the strength of nucleon interactions. The method has great potential but presents new challenges: for theory, the description of the nuclear breakup and final-state interactions; for experiment, the detection of spectator protons, neutrons, and/or other nuclear fragments at very high pseudorapidity.

The tagging method is particularly effective in DIS measurements on the deuteron. The deuteron wave function in nucleonic degrees of freedom $(p n)$ is simple and well known up to nucleon momenta $\approx 300 \mathrm{MeV} / c$ (for the remainder of the article we will set $c=1$ and omit it from the momentum units); non-nucleonic degrees of freedom such as $\Delta$ isobars are suppressed [21]. The detection of the spectator nucleon (proton or neutron) identifies the active nucleon and completely fixes the nuclear configuration in the DIS process. Deuteron DIS with proton spectator tagging at low momenta $p_{p} \lesssim 100 \mathrm{MeV}$ selects DIS events on the neutron in average $p n$ configurations in the deuteron, where some nuclear modifications are present. By performing an extrapolation in the proton spectator momentum one can reach configurations where the nucleons are at asymptotically large separations and effectively free, and in this way extract the free neutron structure function (so-called pole extrapolation) [22-24]. Because of the symmetry between the proton and neutron in the deuteron, one can use the same technique to extract the free proton structure functions with neutron spectator tagging, which allows one to validate the method by comparing with measurements on the proton target. In addition, deuteron DIS with proton or neutron tagging at higher momenta $p_{p, n} \approx$ few $100 \mathrm{MeV}$ selects small-size $p n$ configurations with significant interactions and allows one to study the EMC effect as a function of the configuration size. Other applications include tagged DIS on the polarized deuteron (vector and tensor polarization) [24-26] and tagged diffractive scattering at small $x$ [27,28].

Deuteron DIS with proton tagging was measured in fixedtarget experiments at JLab with $6 \mathrm{GeV}$ electron beam energy using the CLAS spectrometer and the BoNuS proton detector $[29,30]$. The results are used to constrain the $F_{2 n} / F_{2 d}$ structure function ratio at large $x$. Measurements at $12 \mathrm{GeV}$ electron beam energy are planned with the BoNuS and ALERT detectors $[31,32]$. The BoNuS setup detects only protons with momenta $p_{p} \gtrsim 70 \mathrm{MeV}$ (slower protons cannot escape the target), which makes pole extrapolation difficult and requires a model-dependent extraction of free neutron structure. Other DIS experiments with proton and neutron tagging at larger momenta $p_{p, n} \approx$ few $100 \mathrm{MeV}$ explore the EMC effect and its possible connection with nucleon short-range correlations [33-35].

In tagged DIS at the EIC, the spectator nucleon (proton or neutron) from the deuteron breakup emerges in the outgoing ion beam direction, with a momentum given by the boost of its momentum in the deuteron rest frame,

$$
\begin{gathered}
p_{p}(\text { longit }) \approx \frac{p_{d}}{2}\left[1+\frac{p_{p}(\text { longit }, \text { rest frame })}{m_{N}}\right], \\
p_{p}(\text { transv })=p_{p}(\text { transv }, \text { rest frame }),
\end{gathered}
$$

and similarly for $p \rightarrow n$. Here "longitudinal" and "transverse" refer to the outgoing ion beam direction. The spectator longitudinal momentum is given by half the deuteron beam momentum, $p_{d} / 2$, times a factor of order unity determined by the ratio of the longitudinal rest-frame momentum and the nucleon mass $m_{N}$; the spectator transverse momentum is given by the transverse rest-frame momentum. In this kinematics the spectator nucleon can be detected with the far-forward detectors integrated into the outgoing ion beamline [18,19]. The detection of spectator nucleons from nuclear breakup has been a priority of the EIC far-forward detector design since its inception. Protons are detected with a magnetic dipole spectrometer integrated in the first dipole after the interaction point, as well as with Roman pots and off-momentum detectors along the beam path. The setup provides excellent coverage for spectator protons over a broad range of $p_{p}($ longit $) / p_{d} \approx \frac{1}{2}$ and $0<p_{p}$ (transv) $\lesssim 1 \mathrm{GeV}$. Neutrons are detected with a zero-degree calorimeter with comparable coverage. 
The unique far-forward detection capabilities of the EIC, combined with the kinematic coverage for DIS, will enable new types of tagged DIS measurements on the deuteron that have not been possible at previous facilities. Free neutron structure can be extracted through proton tagging with $p_{p}($ transv $) \lesssim 100 \mathrm{MeV}$ and pole extrapolation in the spectator momentum. Free proton structure can be determined through neutron tagging and pole extrapolation, validating the extraction method, since free proton structure can be measured at the EIC in $e p$ collisions in similar kinematics and with the same detector configuration. Nuclear modifications can be studied in detail using both proton and neutron tagging at larger transverse momenta. The physics potential of these measurements calls for a dedicated study. A preliminary assessment of the feasibility and physics impact of tagged DIS at the EIC was made in an earlier Research and Development project [36,37]; this assessment can now be taken to the next level through full detector simulations with the actual EIC far-forward detector design.

In this series of articles we report a comprehensive study of DIS on the deuteron with spectator proton and neutron tagging at the EIC with the baseline far-forward detector design. The objectives are to explore the physics potential of tagged measurements, quantify the detector effects, and provide guidance for optimization of the far-forward detector design. We generate deuteron DIS events using the BeAGLE Monte Carlo (MC) generator [38], reconstruct the spectator nucleon momentum including detector acceptance and resolution and beam-related effects, and perform the physics analysis under realistic conditions. In the present article we study the extraction of free nucleon structure from tagged DIS with pole extrapolation: both free neutron structure from proton tagging and proton structure from neutron tagging. These applications involve far-forward proton and neutron detection at low transverse momenta $p_{p, n}$ (transv) $\lesssim 100 \mathrm{MeV}$, where the acceptance is generally high and uniform, but good momentum resolution is critical. The focus is on studying the performance of the pole extrapolation technique, quantifying the detector resolution effects, and validating the extraction via comparisons with the input model. In a subsequent article we turn to the exploration of nuclear modifications and the tagged EMC effect through tagging at higher transverse momenta $p_{p, n}($ transv $) \approx$ few $100 \mathrm{MeV}$, where the detector acceptance becomes critical [39].

The outline of the article is as follows. In Sec. II we summarize the kinematic variables and experimental observables in tagged DIS, the theoretical description of deuteron structure, and the procedure for free nucleon structure extraction through pole extrapolation. In Sec. III we describe the BeAGLE MC event generator, the EIC far-forward detectors, and the procedure used to quantify the impact of detector acceptance and resolution effects. In Sec. IV we present the steps of the simulated analysis, including the deuteron reduced cross section measurement, the removal of deuteron structure, and the extraction of free nucleon structure through pole extrapolation; we also validate the result of the free nucleon structure extraction by comparing with the model input. In Sec. V we discuss the experimental and theoretical uncertainties of the proposed measurement and explain which of

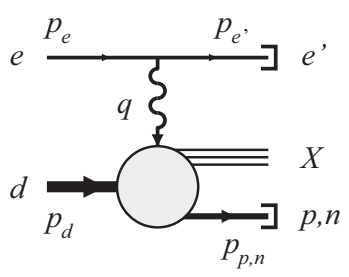

FIG. 1. DIS on the deuteron with detection of a proton (or neutron) in the nuclear fragmentation region, $e+d \rightarrow e^{\prime}+X+p(n)$ ("tagged DIS").

those can be quantified with the present simulations and which require future detailed studies. In Sec. VI we summarize our conclusions. In Sec. VII we discuss possible extensions of the method to other processes of interest.

Appendix A summarizes the deuteron structure model used in the event generation and physics analysis. Appendix B describes the far-forward detector acceptances and resolutions obtained from full simulations, which are used to model the detector response in the present study. These materials can be used in simulations of other nuclear breakup processes at EIC.

\section{PROCESS AND THEORY}

\section{A. Kinematic variables}

We begin by summarizing the variables and observables of tagged DIS measurements, the theoretical description in terms of nuclear and nucleonic structure, and the procedure for extracting free nucleon structure through pole extrapolation. The theoretical framework is described in Refs. [23,24]; here we adapt the formalism to the experimental analysis.

We consider unpolarized inclusive electron scattering on the deuteron, with detection of the scattered electron and an identified proton or neutron in the nuclear fragmentation region (see Fig. 1),

$$
e\left(p_{e}\right)+d\left(p_{d}\right) \rightarrow e^{\prime}\left(p_{e^{\prime}}\right)+X+p\left(p_{p}\right)\left[\text { or } n\left(p_{n}\right)\right] .
$$

The 4-momenta of the particles are denoted as indicated in Eq. (3) and Fig. 1. The 4-momentum transfer is defined as the difference of the initial and final electron 4-momenta,

$$
q \equiv p_{e}-p_{e^{\prime}} .
$$

The DIS process is characterized by the invariant momentum transfer $Q^{2} \equiv-q^{2}$ and the scaling variables

$$
\begin{array}{cl}
x \equiv \frac{Q^{2}}{\left(p_{d} q\right)}, & 0<x<2, \\
y \equiv \frac{\left(p_{d} q\right)}{\left(p_{d} p_{e}\right)}, & 0<y<1,
\end{array}
$$

which satisfy the relation

$$
Q^{2}=\frac{1}{2} x y\left(s_{e d}-M_{d}^{2}\right),
$$

where $s_{e d} \equiv\left(p_{e}+p_{d}\right)^{2}$ is the invariant squared center-ofmass energy of the $e d$ collision and $M_{d}$ is the deuteron mass. The variable $x$ in Eq. (5) is the Bjorken variable computed with $1 / 2$ times the deuteron 4-momentum and can be interpreted as the Bjorken variable for scattering on a nucleon in 
an "unbound" deuteron in which each nucleon carries half the deuteron 4-momentum ( $x$ is a kinematic variable and does not depend on this interpretation; the effects of nuclear binding on the scattering of the nucleon are discussed below). The variable $y$ in Eq. (6) can be interpreted as the fractional energy loss of the electron in the scattering on the deuteron with 4-momentum $p_{d}$, or, equivalently, in the scattering from a nucleon with $p_{d} / 2$ in an unbound deuteron.

We study the process Eq. (3) with proton or neutron detection in the final state. For simplicity we write the following formulas for the case of proton detection; the formulas for neutron detection can be obtained by simple exchange $p \leftrightarrow n$. Situations where additional considerations are needed in obtaining the neutron formulas are indicated in the text.

The momentum of the detected proton (or neutron) in Eq. (3) depends on the reference frame and can be characterized in various ways. For theoretical analysis it is convenient to use a frame in which the momentum transfer $\boldsymbol{q}$ and the deuteron momentum $\boldsymbol{p}_{d}$ are collinear and define the $z$ axis (so-called collinear frame). In this frame one describes the nucleon momentum in terms of its light-front components,

$$
p_{p}^{+} \equiv p_{p}^{0}+p_{p}^{z}, \quad \boldsymbol{p}_{p T} \equiv\left(p_{p}^{x}, p_{p}^{y}\right)
$$

and expresses the proton plus momentum component as a fraction of $1 / 2$ the deuteron plus momentum

$$
p_{p}^{+} \equiv \alpha_{p} p_{d}^{+} / 2, \quad 0<\alpha_{p}<2
$$

the value of $p_{d}^{+}$is arbitrary and can be changed by a boost along the $z$ axis. The light-front variables $\alpha_{p}$ and $\boldsymbol{p}_{p T}$ then characterize the proton momentum in any frame that can be connected to the collinear frame by a Lorentz transformation. Lorentz-invariant expressions of $\alpha_{p}$ and $\boldsymbol{p}_{p T}$, which allow one to compute the variables directly from the 4-vector components of $p_{d}, q$, and $p_{p}$ in any frame without going through a Lorentz transformation, are given in Sec. III H of Ref. [24].

The kinematic limit of $\alpha_{p}$ in the tagged DIS process is dictated by the conservation of light-front plus momentum in the collinear frame,

$$
\alpha_{p}<2(1-\xi) \approx 2(1-x)
$$

where

$$
\xi \equiv \frac{2 x}{1+\sqrt{1+x^{2} M_{d}^{2} / Q^{2}}}=x+O\left(\frac{x^{2} M_{d}^{2}}{Q^{2}}\right)
$$

Equation (10) expresses the fact that the spectator nucleon can have plus momentum at most as large as the total plus momentum of the DIS final state produced on the deuteron (the initial deuteron plus momentum, less the plus momentum removed by the virtual photon). For $x \ll 1$ the upper limit of $\alpha_{p}$ is close to 2 ; for $x \approx 1$ it is significantly below 2 . The invariant phase space element in the spectator momentum is expressed in terms of the variables $\alpha_{p}$ and $\boldsymbol{p}_{p T}$ as

$$
\begin{aligned}
d \Gamma_{p} & \equiv\left[2(2 \pi)^{3}\right]^{-1} \frac{d^{3} p_{p}}{E_{p}}=\left[2(2 \pi)^{3}\right]^{-1} \frac{d \alpha_{p}}{\alpha_{p}} d^{2} p_{p T} \\
& =\left[2(2 \pi)^{3}\right]^{-1} \frac{d \alpha_{p}}{\alpha_{p}} \frac{d p_{p T}^{2}}{2} d \phi_{p},
\end{aligned}
$$

where $p_{p T}^{2} \equiv\left|\boldsymbol{p}_{p T}\right|^{2}$ is the squared modulus and $\phi_{p}$ the azimuthal angle of $\boldsymbol{p}_{p T}$. The case of neutron detection is described by the same formulas with $p \rightarrow n$.

\section{B. Differential cross section}

The basic observable in tagged DIS Eq. (3) is the cross section

$$
d \sigma\left[e d \rightarrow e^{\prime} X p\right] \quad(\text { or } p \rightarrow n),
$$

differential in the momentum of the scattered electron and the observed proton (or neutron); the energy and momentum of the unobserved hadronic final state $X$ follow from 4-momentum conservation and do not count as independent variables. The general structure of the tagged cross section and its parametrization in terms of invariant structure functions are described in Refs. [23,24]. Here we represent the electroproduction cross section (13) in terms of a reduced photoproduction cross section, as is customary in proton electroproduction at HERA; see, e.g., Ref. [40]. In this representation

$$
\begin{aligned}
d \sigma\left[e d \rightarrow e^{\prime} X p\right]= & \operatorname{Flux}\left(x, Q^{2}\right) d x d Q^{2} \frac{d \phi_{e^{\prime}}}{2 \pi} \\
& \times \sigma_{\mathrm{red}, d}\left(x, Q^{2} ; \alpha_{p}, p_{p T}, \phi_{p}\right) d \Gamma_{p} .
\end{aligned}
$$

The first factor represents the virtual photon flux produced by the electron scattering process; it depends only on the electron variables and is differential in $x, Q^{2}$, and the azimuthal angle of the scattered electron around the electron beam direction, $\phi_{e^{\prime}}$. The function is given by

$$
\begin{aligned}
\operatorname{Flux}\left(x, Q^{2}\right) & \equiv \frac{2 \pi \alpha_{\mathrm{em}}^{2} y^{2}}{Q^{4}(1-\epsilon) x} \\
& =\frac{2 \pi \alpha_{\mathrm{em}}^{2}\left[1-(1-y)^{2}\right]}{Q^{4} x}\left[1+O\left(\frac{x^{2} m_{N}^{2}}{Q^{2}}\right)\right],
\end{aligned}
$$

where $\alpha_{\mathrm{em}}$ is the fine structure constant and $\epsilon$ is the virtual photon polarization parameter,

$$
1-\epsilon=\frac{y^{2}}{1+(1-y)^{2}}+O\left(\frac{x^{2} m_{N}^{2}}{Q^{2}}\right)
$$

the exact expression including power corrections can be found in Refs. [23,24]. The flux factor defined in Eq. (15) is identical to the one in electron-nucleon scattering with a nucleon beam of $1 / 2$ the deuteron beam momentum and with $x$ as the standard nucleon Bjorken variable (up to completely negligible kinematic corrections proportional to the deuteron binding energy); this definition allows for an easy comparison with the formulas and results in electron-proton scattering at HERA. 
The second factor in Eq. (14) represents the reduced cross section for tagged deuteron DIS; it depends on both the electron variables $x, Q^{2}$ and the tagged nucleon variables $\alpha_{p}, p_{p T}$, and $\phi_{p}$, and is proportional to the differential phase space of the tagged nucleon momentum, $d \Gamma_{p}$ in Eq. (12). The function $\sigma_{\text {red, } d}$ contains the hadronic information in the tagged DIS cross section. Its dependence on $y$ (or $\epsilon$ ) and on $\phi_{p}$ is dictated by relativistic covariance and can be made explicit by expanding it in structure functions, ${ }^{1}$

$$
\begin{aligned}
\sigma_{\text {red }, d} & \left(x, Q^{2} ; \alpha_{p}, p_{p T}, \phi_{p}\right) \\
= & F_{2 d}\left(x, Q^{2} ; \alpha_{p}, p_{p T}\right)-(1-\epsilon) F_{L d}\left(x, Q^{2} ; \alpha_{p}, p_{p T}\right) \\
& +\phi_{p} \text {-dependent structures. }
\end{aligned}
$$

The tagged structure functions $F_{2 d}$ and $F_{L d}$ depend on the tagged proton momentum only through the light-front fraction $\alpha_{p}$ and the transverse momentum modulus $p_{p T}$. Equation (17) presents only the terms in the reduced cross section that do not explicitly depend on $\phi_{p}$; the full structure including the $\phi_{p}$ dependence is given in Ref. [41]. In the analysis performed here we consider only the cross section averaged over $\phi_{p}$, in which the $\phi_{p}$ dependent structures average to zero:

$$
\begin{aligned}
& \bar{\sigma}_{\text {red }, d}\left(x, Q^{2} ; \alpha_{p}, p_{p T}\right) \\
& \quad \equiv \int \frac{d \phi_{p}}{2 \pi} \sigma_{\text {red }, d}\left(x, Q^{2} ; \alpha_{p}, p_{p T}, \phi_{p}\right) \\
& \quad=F_{2 d}\left(x, Q^{2} ; \alpha_{p}, p_{p T}\right)-(1-\epsilon) F_{L d}\left(x, Q^{2} ; \alpha_{p}, p_{p T}\right) .
\end{aligned}
$$

The case of neutron tagging is described by the same formulas with $p \rightarrow n$.

The tagged deuteron structure functions $F_{2 d}, F_{L d}$, etc. contain the basic information that can be extracted from tagged DIS measurements. They represent a special case of semiinclusive DIS structure functions [8], with the target being the deuteron nucleus and the observed hadron being a nucleon in the nuclear fragmentation region. Note that no assumptions regarding a composite nuclear structure in terms of nucleons or a particular reaction mechanism are made in the general decomposition of Eqs. (17) and (18). A relation between the tagged deuteron structure functions and the neutron structure functions $F_{2 n}$ and $F_{L n}$ (or the proton structure functions in the case of neutron tagging) can be established only in the context of a theoretical description combining nuclear and nucleonic structure.

\section{Deuteron structure description}

The theoretical treatment of tagged DIS starts from the picture of the nucleus as a composite system of nucleons and describes the cross section by combining nuclear and nucleonic structure. The objectives are to predict the tagged structure functions in terms of the nucleon structure functions and calculable nuclear structure elements, and to enable the

${ }^{1}$ The $F_{L d}$ structure function in Eq. (17) is defined as in Ref. [40] and differs from the one in Ref. [24] by a factor $x: F_{L d}$ (here) $=$ $x F_{L d}$ (Ref. [24]). extraction of the nucleon structure functions from the tagged DIS data.

Nuclear binding modifies the deep-inelastic structure of the nucleus relative to the sum of free nucleons in several ways: (i) The motion of the nucleons in the nucleus shifts the effective kinematics in the electron-nucleon scattering process. (ii) The interactions between the nucleons affect the partonic structure seen by the high-energy probe. These effects can be interpreted alternatively as a modification of bound nucleon structure or the presence of non-nucleonic degrees of freedom and have been the object of extensive studies; see Refs. [11-14,42] for a review. In the present study of tagged DIS, the interaction effects are eliminated by pole extrapolation, which selects large-size $p n$ configurations in the deuteron where the nucleons are effectively free. This avoids the need for an explicit description of these effects and greatly simplifies the theoretical treatment.

The nucleonic structure of the deuteron is described at fixed light-front time $x^{+} \equiv t+z$ (light-front quantization). This quantization scheme is unique in the sense that the energy off-shellness of the electron-nucleon scattering subprocess (the energy difference between the initial and final state) remains finite in the limit of large incident energy, so that one can describe the electron-nucleon subprocess in terms of the on-shell scattering amplitude and construct a composite description [21]. The nucleon 4-momenta in the deuteron are characterized by their "plus" and transverse components in the collinear frame [see Sec. II A and Eqs. (8) and (9)]:

$$
\begin{aligned}
& p_{p}^{+}=\alpha_{p} p_{d}^{+} / 2, \quad \boldsymbol{p}_{p T}, \\
& p_{n}^{+}=\left(2-\alpha_{p}\right) p_{d}^{+} / 2, \quad \boldsymbol{p}_{n T}=-\boldsymbol{p}_{p T} ;
\end{aligned}
$$

the proton and neutron momenta are related by light-front momentum conservation (the transverse momentum of the deuteron bound state in the collinear frame is zero, $\boldsymbol{p}_{d T}=0$ ). The "minus" components of the 4-momenta play the role of energies and are fixed by the mass-shell conditions $p_{p, n}^{2}=m_{N}^{2}$,

$$
\begin{aligned}
& p_{p}^{-}=\left(\left|\boldsymbol{p}_{p T}\right|^{2}+m_{N}^{2}\right) / p_{p}^{+}, \\
& p_{n}^{-}=\left(\left|\boldsymbol{p}_{n T}\right|^{2}+m_{N}^{2}\right) / p_{n}^{+} .
\end{aligned}
$$

Here $m_{N} \equiv\left(m_{p}+m_{n}\right) / 2$ denotes the average nucleon mass; we assume isospin symmetry and neglect the difference of proton and neutron masses; see Appendix A 2. The superposition of $p n$ configurations in the deuteron is described by the light-front wave function

$$
\Psi_{d}\left(\alpha_{p}, \boldsymbol{p}_{p T}\right),
$$

which is normalized such that

$$
\int \frac{d \alpha_{p} d^{2} p_{p T}}{\alpha_{p}\left(2-\alpha_{p}\right)}\left|\Psi_{d}\left(\alpha_{p}, \boldsymbol{p}_{p T}\right)\right|^{2}=1 .
$$

Here we suppress the nucleon and deuteron spin variables for brevity; the full expressions including spins are given in Appendix A 3 and Ref. [24]. The deuteron light-front wave function Eq. (23) can be obtained by solving the lightfront bound state equation with realistic $p n$ interactions [21]. In the present study we use an approximation where the light-front wave function is constructed from the well-known 


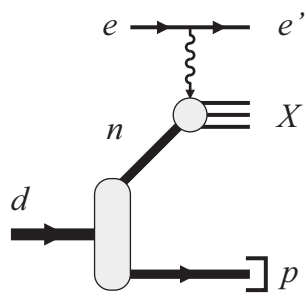

(a)

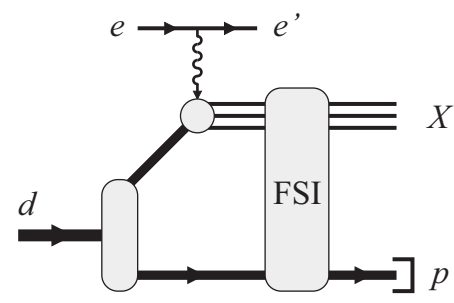

(b)
FIG. 2. Theoretical description of tagged DIS. (a) Impulse approximation. (b) Final-state interactions.

nonrelativistic wave function of the deuteron bound state; see Appendix A 4. This approximation is accurate at nucleon restframe momenta $\left|\boldsymbol{p}_{p, n}\right| \lesssim 100 \mathrm{MeV}$ used in low-momentum tagging. In particular, the approximation correctly implements the analytic properties of the deuteron wave function and the "nucleon pole" used in the extraction of free nucleon structure with pole extrapolation; see Appendix A 5. Altogether, the deuteron light-front structure is theoretically well understood and can reliably be constructed in the momentum range probed in the present study. The tagged DIS cross section is calculated in the impulse approximation [see Fig. 2(a)]. It takes into account the motion of the active nucleon in the deuteron and its correlation with the spectator kinematics as governed by the deuteron wave function, but does not include dynamical initial-state modifications or final-state interactions [Fig. 2(b)]. In the impulse approximation the tagged deuteron structure functions for the case of proton tagging are obtained as $[23,24]$

$$
\begin{aligned}
& F_{2 d}(\left.x, Q^{2} ; \alpha_{p}, p_{p T}\right) \\
&= {\left[2(2 \pi)^{3}\right] \mathcal{S}_{d}\left(\alpha_{p}, p_{p T}\right) F_{2 n}\left(x_{n}, Q^{2}\right) } \\
&+ \text { initial-state modifications } \\
&+ \text { final-state interactions, } \\
& F_{L d}\left(x, Q^{2} ; \alpha_{p}, p_{p T}\right) \\
&=\left[2(2 \pi)^{3}\right] \mathcal{S}_{d}\left(\alpha_{p}, p_{p T}\right) F_{L n}\left(x_{n}, Q^{2}\right) \\
& \quad+\text { initial-state modifications } \\
& \quad+\text { final-state interactions. }
\end{aligned}
$$

Here $\mathcal{S}_{d}$ is the deuteron light-front spectral function. It depends on the tagged proton momentum variables $\alpha_{p}$ and $p_{p T} \equiv\left|\boldsymbol{p}_{p T}\right|$ (unpolarized deuteron) and represents the density of the deuteron light-front wave function times a flux factor depending on $\alpha_{p}$,

$$
\mathcal{S}_{d}\left(\alpha_{p}, p_{p T}\right) \equiv \frac{\left|\Psi_{d}\left(\alpha_{p}, \boldsymbol{p}_{p T}\right)\right|^{2}}{2-\alpha_{p}} .
$$

$F_{2 n}$ and $F_{L n}$ are the DIS structure functions of the neutron. They are evaluated at the effective scaling variable

$$
x_{n} \equiv \frac{x}{2-\alpha_{p}},
$$

which results from the fact that the neutron plus momentum in the deuteron is determined by that of the spectator proton; see Eq. (20). This shows how detection of the spectator fixes the nuclear configuration in the DIS process. The momentum transfer $Q^{2}$ in the neutron structure functions is equal to the electron variable $Q^{2}$. The quoted expressions for the neutron structure function arguments $x_{n}$ and $Q^{2}$ are valid up to power corrections $\approx m_{N}^{2} / Q^{2}$, which are negligible in our kinematics.

From the impulse approximation results for the tagged structure functions we obtain the tagged reduced cross section as

$$
\begin{aligned}
\bar{\sigma}_{\text {red }, d} & \left(x, Q^{2} ; \alpha_{p}, p_{p T}\right) \\
= & {\left[2(2 \pi)^{3}\right] \mathcal{S}_{d}\left(\alpha_{p}, p_{p T}\right) \sigma_{\text {red }, n}\left(x_{n}, Q^{2}\right) } \\
& + \text { initial-state modifications } \\
& + \text { final-state interactions, }
\end{aligned}
$$

where $\sigma_{\text {red, } n}$ is the reduced cross section for DIS on the neutron,

$$
\sigma_{\text {red }, n}\left(x_{n}, Q^{2}\right)=F_{2 n}\left(x_{n}, Q^{2}\right)-(1-\epsilon) F_{L n}\left(x_{n}, Q^{2}\right) .
$$

Here we have used the fact that the $\epsilon$ parameter for scattering on the deuteron (with scaling variable $x$ ) is equal to that for scattering on the neutron (with $x_{n}$ ) up to power corrections $\propto y^{2} m_{N}^{2} / Q^{2}$, which can be neglected in DIS kinematics. Equation (29) concisely summarizes the impulse approximation result as relevant to unpolarized tagged DIS without longitudinal/transverse separation.

The case of neutron tagging is described by analogous formulas. The reduced deuteron cross section for neutron tagging in the impulse approximation is

$$
\begin{aligned}
& \bar{\sigma}_{\text {red }, d}\left(x, Q^{2} ; \alpha_{n}, p_{n T}\right) \\
& \quad=\left[2(2 \pi)^{3}\right] \mathcal{S}_{d}\left(\alpha_{n}, p_{n T}\right) \sigma_{\text {red }, p}\left(x_{p}, Q^{2}\right)+\bmod .,
\end{aligned}
$$

where the spectral function is now

$$
\mathcal{S}_{d}\left(\alpha_{n}, p_{n T}\right) \equiv \frac{\left|\Psi_{d}\left(\alpha_{n}, \boldsymbol{p}_{n T}\right)\right|^{2}}{2-\alpha_{n}},
$$

and the reduced proton cross section is

$$
\sigma_{\text {red }, p}\left(x_{p}, Q^{2}\right)=F_{2 p}\left(x_{p}, Q^{2}\right)-(1-\epsilon) F_{L p}\left(x_{p}, Q^{2}\right),
$$

evaluated at the effective scaling variable

$$
x_{p} \equiv \frac{x}{2-\alpha_{n}} .
$$

The spectral function for neutron tagging, Eq. (32), is given by the same mathematical function as for proton tagging, Eq. (27), only evaluated at the neutron momentum variables $\alpha_{n}$ and $\boldsymbol{p}_{n T}$. The symmetry properties of the deuteron light-front wave function and spectral function under protonneutron interchange are summarized in Appendix A 2; see in particular Eqs. (A11) and (A15).

\section{Nucleon structure extraction}

In the present work we study the extraction of free nucleon structure from tagged DIS measurements. This requires separating deuteron and nucleon structure in the measured 
cross section, and-if possible-suppressing the effects of initial-state modifications and final-state interactions. This can be accomplished using the dependence of the tagged cross section on the spectator nucleon momentum. In the following we discuss two methods:

Method I: Integration over spectator momentum. This method uses proton tagging only to identify events with an active neutron, but does not measure the spectator momentum, so it integrates over the spectator or active nucleon kinematics. Initial-state modifications of neutron structure are not suppressed; their strength is comparable to that in inclusive nuclear DIS. This is the traditional method for tagged DIS analysis. The overall uncertainty is dominated by the unknown nuclear modifications. We simulate such measurements with the EIC only as a reference point, to enable comparisons with other methods and internal validation.

Method II: Pole extrapolation in spectator momentum. This method uses the analytic properties of the deuteron wave function to select large-size $p n$ configurations in the deuteron, in which both initial-state modifications and final-state interactions are suppressed. It enables a model-independent extraction of free neutron structure. The resulting uncertainty is determined by the quality of the measurement and the extrapolation procedure. This novel method demands good detector coverage and resolution at small proton momenta $\left|\boldsymbol{p}_{p T}\right| \ll 100 \mathrm{MeV}$ and may become possible with the EIC. We simulate such measurements with the EIC as the potential method of choice for free neutron structure extraction with proton tagging (and proton structure with neutron tagging) and quantify its uncertainties.

\section{E. Integration over spectator momentum}

Taking the impulse approximation expressions of the tagged deuteron structure functions, Eqs. (25) and (26), and computing the integral over the spectator momentum, we obtain

$$
\begin{aligned}
& \int d \Gamma_{p} F_{2 d}\left(x, Q^{2} ; \alpha_{p}, p_{p T}\right) \\
= & \int \frac{d \alpha_{p}}{\alpha_{p}} d^{2} p_{p T} \mathcal{S}_{d}\left(\alpha_{p}, p_{p T}\right) F_{2 n}\left(x_{n}, Q^{2}\right) \\
\approx & F_{2 n}\left(x, Q^{2}\right) \int \frac{d \alpha_{p}}{\alpha_{p}} d^{2} p_{p T} \mathcal{S}_{d}\left(\alpha_{p}, p_{p T}\right) \\
= & F_{2 n}\left(x, Q^{2}\right)
\end{aligned}
$$

and similarly for $F_{L d}$ and $F_{L n}$. The expressions of Eq. (35) are valid up to initial-state modifications of nucleon structure, which are not included in the impulse approximation. In Eq. (35a) we have used the explicit form of the proton phase space element $d \Gamma_{p}$ in terms of the light-front momentum variables $\alpha_{p}$ and $\boldsymbol{p}_{p T}$, Eq. (12). In Eq. (35a) the variable $x_{n}$ in the argument of $F_{2 n}$ depends on the integration variable $\alpha_{p}$, so that the integral represents a convolution of the spectral function and the neutron structure function. The integrand is concentrated around $\alpha_{p}=1$ because of the shape of the spectral function. Equation (35b) is obtained in the "peaking approximation," where one neglects the $\alpha_{p}$ dependence of $F_{2 n}$ under the integral and approximates

$$
F_{2 n}\left(x_{n}, Q^{2}\right) \approx F_{2 n}\left(x, Q^{2}\right) .
$$

Equation (35c) is then obtained by using the integral relation ("sum rule") of deuteron spectral function Eq. (27),

$$
\int \frac{d \alpha_{p}}{\alpha_{p}} d^{2} p_{p T} \mathcal{S}_{d}\left(\alpha_{p}, p_{p T}\right)=1,
$$

which follows from the normalization condition of the deuteron light-front wave function Eq. (24).

Combining the relations of Eq. (35) for $F_{2 d}$ and $F_{L d}$, we find that the integral of the reduced tagged cross section from Eq. (17) over the spectator momentum is equal to the reduced cross section for scattering on the neutron:

$$
\begin{aligned}
& \int d \Gamma_{p} \sigma_{\text {red }, d}\left(x, Q^{2} ; \alpha_{p}, p_{p T}, \phi_{p}\right) \\
& \approx F_{2 n}\left(x, Q^{2}\right)-(1-\epsilon) F_{2 L}\left(x, Q^{2}\right) \\
& \quad \equiv \sigma_{\text {red }, n}\left(x, Q^{2}\right) .
\end{aligned}
$$

Including the flux factor Eq. (15), we see that the tagged electron-deuteron cross section in the impulse approximation, integrated over the spectator momentum, is equal to the electron-neutron cross section in nominal kinematics (neutron with $1 / 2$ the deuteron beam momentum; see Sec. II B)

$$
\begin{aligned}
d \sigma[e d & \left.\left.\rightarrow e^{\prime} X p \text { (integrated }\right)\right] \\
= & \operatorname{Flux}\left(x, Q^{2}\right) d x d Q^{2} \frac{d \phi_{e^{\prime}}}{2 \pi} \\
& \times \int d \Gamma_{p} \sigma_{\text {red }, d}\left(x, Q^{2} ; \alpha_{p}, p_{p T}, \phi_{p}\right) \\
= & \operatorname{Flux}\left(x, Q^{2}\right) d x d Q^{2} \frac{d \phi_{e^{\prime}}}{2 \pi} \times \sigma_{\mathrm{red}, n}\left(x, Q^{2}\right) .
\end{aligned}
$$

Equation (39) provides a simple connection between the tagged deuteron and neutron cross sections in the impulse approximation. Its value for neutron structure extraction is limited by the fact that it is specific to the impulse approximation and does not include initial-state modifications, which are generally as large as in untagged inclusive scattering. However, in simulations with an impulse-approximation-based physics model, Eq. (39) serves as a simple way of recovering the neutron structure input, and we use it in this sense in our validation in Sec. IV D.

\section{F. Pole extrapolation in spectator momentum}

The deuteron light-front wave function is an analytic function of the nucleon momentum variables. It can be considered both at physical (real) and unphysical (imaginary) values of the momentum, and its behavior is governed by singularities in the unphysical region. The dominant feature at low momenta is the "nucleon pole" singularity, which results from the free motion of the nucleons outside the range of the nucleonnucleon interactions. It is of the form [24]

$$
\Psi_{d}\left(\alpha_{p}, \boldsymbol{p}_{p T}\right)=\frac{R}{p_{p T}^{2}+a_{T}^{2}}+\text { (less singular) }
$$


The pole in $p_{p T}^{2} \equiv\left|\boldsymbol{p}_{p T}\right|^{2}$ occurs at $p_{p T}^{2}=-a_{T}^{2}<0$ in the unphysical region. The position is given by the squared transverse mass of the $p n$ configuration,

$$
a_{T}^{2} \equiv a_{T}^{2}\left(\alpha_{p}\right)=\left(\alpha_{p}-1\right)^{2} m_{N}^{2}+\alpha_{p}\left(2-\alpha_{p}\right) a^{2},
$$

where

$$
a^{2} \equiv m_{N} \epsilon_{d}
$$

in which $\epsilon_{d}$ is the deuteron binding energy. $a^{2}$ defines the position of the nucleon pole in the nonrelativistic wave function and provides a measure of the natural size of the deuteron (see Appendix A 5). The transverse mass (41) depends on $\alpha_{p}$ and attains its minimal value $a_{T}^{2}=a^{2}$ at $\alpha_{p}=1$. The residue of the pole in Eq. (40) is given by

$$
R \equiv R\left(\alpha_{p}\right) \equiv \alpha_{p}\left(2-\alpha_{p}\right) \sqrt{m_{N}} \Gamma,
$$

where $\Gamma$ is the residue of the nucleon pole of the nonrelativistic deuteron wave function (see Appendix A 5).

The nucleon pole singularity in the deuteron light-front wave function (40) has a simple physical interpretation. In the transverse coordinate representation of the wave function, it describes $p n$ configurations with asymptotically large transverse size $r_{T} \rightarrow \infty$ in the deuteron [24]. At such distances the nucleons are outside of the range of the nucleon-nucleon interactions, and their motion is essentially free. The nucleon pole thus represents a universal feature of the deuteron as a weakly bound system. It can be derived from the structure of the bound state equation and is found in all models that describe the deuteron as a bound state with a finite-range nucleon-nucleon interaction. The pole position (41) follows from kinematic considerations and is known exactly. The residue (43) can be inferred from non-relativistic deuteron structure calculations and low-energy measurements and is known with an accuracy $\lesssim 1 \%$ (see Appendix A 5 and Table II).

Tagged DIS at physical transverse momenta $p_{p T}^{2}>0$ always samples finite-size $p n$ configurations in the deuteron, where nucleon interactions are generally present. However, analytic continuation to unphysical momenta $p_{p T}^{2} \rightarrow-a_{T}^{2}$ can effectively access infinite-size configurations $r_{T} \rightarrow \infty$, where nucleon interactions are absent. Final-state interactions of the DIS products with the spectator are also suppressed in such configurations. This allows one to practically realize DIS on an unbound nucleon in the deuteron and to extract free neutron structure. In the light-front spectral function (27), the nucleon pole (40) gives rise to a singularity of the form,

$$
\begin{aligned}
\mathcal{S}_{d}\left(\alpha_{p}, p_{p T}\right) & \left.=\frac{C}{\left(p_{p T}^{2}+a_{T}^{2}\right)^{2}}+\text { (less singular }\right) \\
& \equiv \mathcal{S}_{d}\left(\alpha_{p}, p_{p T}\right)[\text { pole }]
\end{aligned}
$$

where the residue is

$$
C \equiv C\left(\alpha_{p}\right) \equiv \alpha_{p}^{2}\left(2-\alpha_{p}\right) m_{N} \Gamma^{2} .
$$

The nucleon pole (44) dominates the behavior of the spectral function at low transverse momenta in the physical region. Figure 3(a) shows the spectral function and its pole term as functions of $p_{p T}^{2}$ in the physical $\left(p_{p T}^{2}>0\right)$ and unphysical
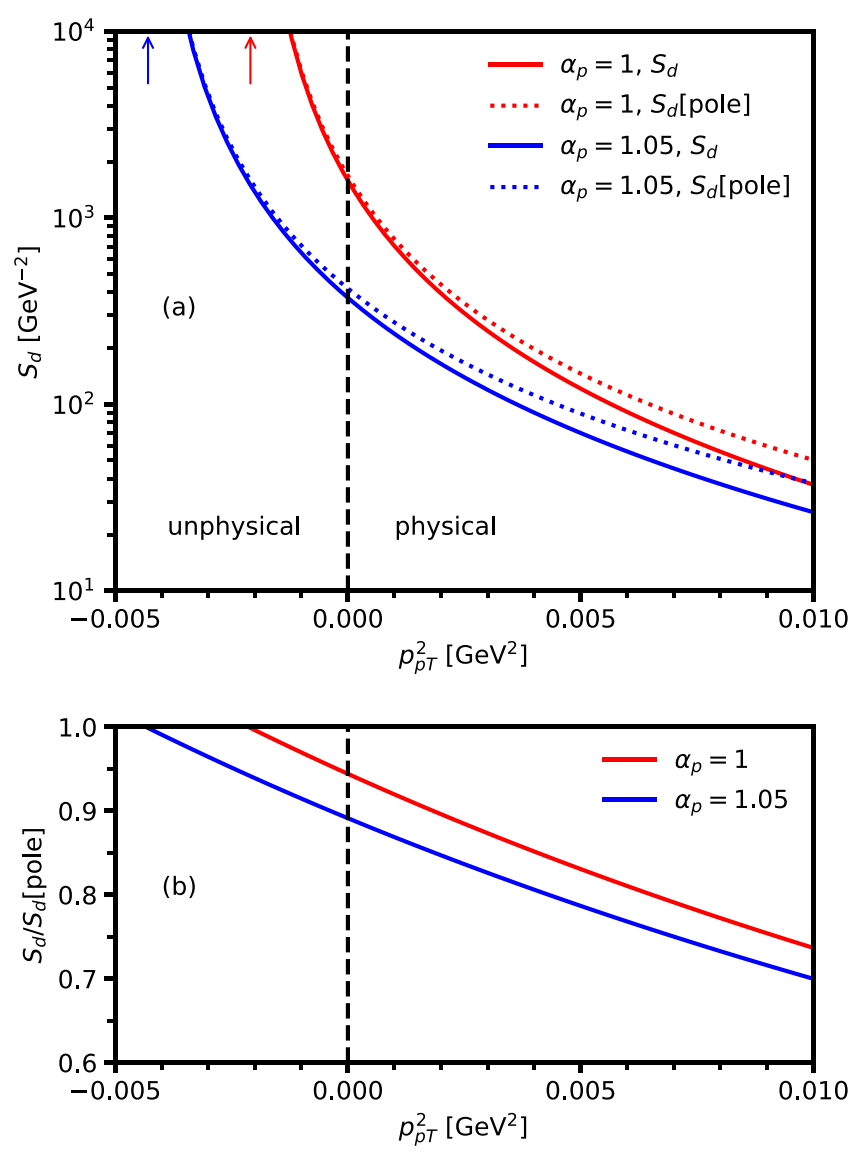

FIG. 3. Deuteron spectral function and its nucleon pole contribution. (a) Spectral function $\mathcal{S}_{d}\left(\alpha_{p}, p_{p T}\right)$, Eq. (27) (solid lines), and its pole term $\mathcal{S}_{d}\left(\alpha_{p}, p_{p T}\right)$ [pole], Eq. (44) (dashed lines), as a function of $p_{p T}^{2}$, for two fixed values of $\alpha_{p}$. The plot shows the functions in the physical $\left(p_{p T}^{2}>0\right)$ and unphysical regions $\left(p_{p T}^{2}<0\right)$. The positions of the poles at $p_{p T}^{2}=-a_{T}^{2}\left(\alpha_{p}\right)$ are marked by arrows for the two values of $\alpha_{p}$. (b) Ratio of the full spectral function and the pole term, Eq. (46), as a function of $p_{p T}^{2}$, for the same fixed values of $\alpha_{p}$.

regions $\left(p_{p T}^{2}<0\right)$, for fixed values of $\alpha_{p}$. (This numerical example uses the two-pole parametrization of the wave function of Appendix A 6.) One observes that the pole term accounts for most of the value and the variation of the spectral function in the physical region $0<p_{p T}^{2} \lesssim 0.01 \mathrm{GeV}^{2}$; the spectral function varies by an order of magnitude over this interval. Figure 3(b) shows the ratio of the spectral function and the pole term,

$$
\frac{\mathcal{S}_{d}\left(\alpha_{p}, p_{p T}\right)}{\mathcal{S}_{d}\left(\alpha_{p}, p_{p T}\right)[\mathrm{pole}]},
$$

as a function of $p_{p T}^{2}$ in the same interval. One notes that the deviations of the full spectral function from the pole term are $\lesssim 30 \%$ for $0<p_{p T}^{2} \lesssim 0.01 \mathrm{GeV}^{2}$, and that dividing the full spectral function by the pole term removes most of the $p_{p T}^{2}$ dependence. In particular, the plots also illustrate that, when following the dependence into the unphysical region $p_{p T}^{2}<0$ and approaching the pole at $p_{p T}^{2} \rightarrow-a_{T}^{2}$, the pole term represents the entire spectral function, as implied by 
Eq. (44), and the ratio becomes unity,

$$
\frac{\mathcal{S}_{d}\left(\alpha_{p}, p_{p T}\right)}{\mathcal{S}_{d}\left(\alpha_{p}, p_{p T}\right)[\mathrm{pole}]} \rightarrow 1 \quad\left(p_{p T}^{2} \rightarrow-a_{T}^{2}\right) .
$$

The existence of the nucleon pole and its properties enable a unique method for neutron structure extraction from DIS on the deuteron with proton tagging ("pole extrapolation"):

(i) Measure the tagged DIS cross section Eq. (14) at fixed $\alpha_{p}$ and small physical transverse momenta, remove the flux factor, and extract the $\phi_{p}$-integrated reduced cross section:

$$
\begin{aligned}
& \sigma_{\text {red }, d}\left(x, Q^{2} ; \alpha_{p}, p_{p T}, \phi_{p}\right) \\
& \quad=\left[\operatorname{Flux}\left(x, Q^{2}\right)\right]^{-1} \frac{d \sigma\left[e d \rightarrow e^{\prime} X p\right]}{d x d Q^{2}\left(d \phi_{e^{\prime}} / 2 \pi\right) d \Gamma_{p}}, \\
& \bar{\sigma}_{\text {red }, d}\left(x, Q^{2} ; \alpha_{p}, p_{p T}\right) \\
& \quad=\int \frac{d \phi_{p}}{2 \pi} \sigma_{\text {red }, d}\left(x, Q^{2} ; \alpha_{p}, p_{p T}, \phi_{p}\right) .
\end{aligned}
$$

(ii) Divide the reduced cross section by the theoretically known pole term of the spectral function, Eq. (44):

$$
\frac{\bar{\sigma}_{\text {red }, d}\left(x, Q^{2} ; \alpha_{p}, p_{p T}\right)}{\mathcal{S}_{d}\left(\alpha_{p}, p_{p T}\right)[\text { pole }]} .
$$

In the impulse approximation, Eqs. (25) and (26), this ratio is theoretically equal to

$$
\begin{aligned}
{[\cdots]=} & \frac{\mathcal{S}_{d}\left(\alpha_{p}, p_{p T}\right) \sigma_{\text {red }, n}\left(x_{n}, Q^{2}\right)}{\mathcal{S}_{d}\left(\alpha_{p}, p_{p T}\right)[\text { pole }]} \\
& + \text { initial-state modifications } \\
& + \text { final-state interactions. }
\end{aligned}
$$

(iii) Extrapolate the ratio (50) in $p_{p T}^{2}$ to the point $p_{p T}^{2}=$ $-a_{T}^{2}$ (pole position) in the unphysical region by a loworder polynomial fit:

$$
\begin{aligned}
& \sigma_{\text {red }, n}\left(x_{n}, Q^{2}\right) \\
& \quad=\lim \left[p_{p T}^{2} \rightarrow-a_{T}^{2}\right] \frac{\bar{\sigma}_{\text {red }, d}\left(x, Q^{2} ; \alpha_{p}, p_{p T}\right)}{\mathcal{S}_{d}\left(\alpha_{p}, p_{p T}\right)[\text { pole }]} .
\end{aligned}
$$

At the pole both initial-state modifications and finalstate interactions in Eq. (51) vanish, and the impulse approximation becomes exact [22,23]. Furthermore, at the pole the ratio of the full spectral function to the pole term becomes unity, Eq. (47). The procedure (52) therefore returns the free neutron reduced cross section without nuclear modifications.

The theoretical uncertainties arising in the pole extrapolation and the role of model dependence in the physical region are discussed in Sec. V B.

\section{SIMULATION TOOLS AND DETECTORS}

\section{A. BeAGLE Monte Carlo generator}

We now describe the simulation tools used in the present study, the EIC far-forward detector design, and the specific considerations in the reconstruction of the far-forward spectator momentum.

BeAGLE is a general purpose lepton-nucleus $(e A)$ event generator, which combines PYTHIA 6.4 [43], DPMJET 3.0 [44], and the FLUKA model $[45,46]$. A detailed description of the entire program can be found in Ref. [38]. In the modeling of scattering on the deuteron and other light ions, DPMJET and FLUKA are not used, and the high-energy scattering process is treated in the impulse approximation (no final-state interactions). The parts of BeAGLE used in the present analysis are the electron-nucleon DIS process modeled by PYTHIA 6.4 and the deuteron light-front spectral function describing the kinematic distribution of the spectator nucleon; see Sec. II C, Appendix A, and Ref. [47]. The generator thus implements the theoretical framework for tagged deuteron DIS as described in Sec. II and can be used for simulations of nucleon structure extraction with pole extrapolation. The present version of BeAGLE uses the parametrization of Ref. [48] to generate the deuteron spectral function; the nucleon pole parameters for this parametrization are given in Appendix A 5 and Table II. The code version (git tag) used in the present study is BeAGLE 1.01.03; the deuteron structure implementation in this version is the same as in BeAGLE 1.0 used in Ref. [47].

BeAGLE describes electron-neutron scattering in the same way as electron-proton scattering in PYTHIA 6, adjusting for the different isospin in the initial state (different proton and neutron PDFs). For technical reasons the proton and neutron PDFs in the generator include an empirical nuclear modification modeled on that of the alpha particle $A=4$ [38]. This feature is irrelevant for the present study and does not affect the results, as we look at the extracted neutron and proton structure functions only relative to the model input, not in absolute terms. Because BeAGLE describes electron-deuteron scattering in the impulse approximation, it gives the same result when extracting nucleon structure using integration over the spectator momentum or pole extrapolation (Methods I and II of Sec. IIE). We use this feature to validate the results of the pole extrapolation simulations in Sec. IV D.

The treatment of the kinematics of the electron-nucleon scattering process in BeAGLE requires some explanation. For technical reasons BeAGLE evaluates the electron-nucleon DIS cross section at the Bjorken variable of the unbound nucleon, $x$, Eq. (5), not at the effective variable of the bound nucleon, $x_{n}$, Eq. (28). The tagged DIS cross section used in BeAGLE therefore differs from the true impulse approximation value by the factor (for events with tagged proton and active neutron)

$$
\frac{\sigma\left[e n \rightarrow e^{\prime} X\right]\left(x, Q^{2}\right)}{\sigma\left[e n \rightarrow e^{\prime} X\right]\left(x_{n}, Q^{2}\right)}
$$

where $\sigma\left[e n \rightarrow e^{\prime} X\right]$ is the electron-neutron DIS cross section. This difference needs to be taken into account in simulations of cross section measurements with BeAGLE. It can easily be corrected by multiplying the cross sections extracted from BeAGLE with a correction factor given by the inverse of 
Eq. (53):

$$
\begin{aligned}
\operatorname{Corr}\left(x, Q^{2} ; \alpha_{p}\right) & \equiv \frac{\sigma\left[e n \rightarrow e^{\prime} X\right]\left(x_{n}, Q^{2}\right)}{\sigma\left[e n \rightarrow e^{\prime} X\right]\left(x, Q^{2}\right)} \\
& =\frac{\operatorname{Flux}\left(x_{n}, Q^{2}\right) \sigma_{\text {red }, n}\left(x_{n}, Q^{2}\right)}{\operatorname{Flux}\left(x, Q^{2}\right) \sigma_{\text {red }, n}\left(x, Q^{2}\right)} \\
& =\frac{x \sigma_{\text {red }, n}\left(x_{n}, Q^{2}\right)}{x_{n} \sigma_{\text {red }, n}\left(x, Q^{2}\right)} \\
& =\left(2-\alpha_{p}\right) \frac{\sigma_{\text {red }, n}\left(x_{n}, Q^{2}\right)}{\sigma_{\text {red }, n}\left(x, Q^{2}\right)} .
\end{aligned}
$$

In Eq. (54b) we have expressed the electron-neutron DIS cross sections in terms of the flux factors (15) and the reduced cross sections (30); in Eq. (54c) we have used that the flux factor at fixed $Q^{2}$ is proportional to $1 / x$; in Eq. (54d) we have replaced the ratio $x / x_{n}$ by $2-\alpha_{p}$ using Eq. (28). Thus the correction factor is given by a simple expression in terms of the tagged proton $\alpha_{p}$ and the neutron reduced cross section ratio.

The correction factor (54) satisfies

$$
\operatorname{Corr}\left(x, Q^{2} ; \alpha_{p}=1\right) \equiv 1,
$$

because $x_{n}=x$ at $\alpha_{p}=1$; see Eq. (28). For $\left|1-\alpha_{p}\right| \ll 1$ we can expand the factor around $\alpha_{p}=1$ and obtain

$$
\begin{aligned}
\operatorname{Corr}\left(x, Q^{2} ; \alpha_{p}\right) & =1+\left[1-\frac{x \frac{d}{d x} \sigma_{\text {red }, n}\left(x, Q^{2}\right)}{\sigma_{\text {red }, n}\left(x, Q^{2}\right)}\right]\left(1-\alpha_{p}\right) \\
& \approx 1+(1+\lambda)\left(1-\alpha_{p}\right) .
\end{aligned}
$$

In Eq. (56a) the first term in the bracket, 1, is the "kinematic" correction resulting from the flux factors; the second term is the "dynamical" correction resulting from the reduced cross sections. The form of Eq. (56b) applies at $x \ll 0.1$, where the reduced cross section depends on $x$ approximately as $\sigma_{\text {red, } n}\left(x, Q^{2}\right) \propto x^{-\lambda}$, with $\lambda \equiv \lambda\left(Q^{2}\right)$ [40]. The HERA measurements find values $\lambda \approx 0.15-0.2$ [40], showing that the dynamical correction is small and the kinematic correction dominates at $x \ll 0.1$. Note that the dynamical correction is generally large at $x \gtrsim 0.1$, where the nucleon structure functions and the reduced cross section strongly depend on $x$.

\section{B. Kinematics and event sample}

In the present study we use the EIC configuration with $18 \mathrm{GeV}$ electrons colliding with $110 \mathrm{GeV} /$ nucleon deuterons, corresponding to an electron-nucleon squared center-of-mass energy of $s_{e N} \equiv s_{e d} / 2=(89 \mathrm{GeV})^{2}$. The simulations can easily be adapted to other beam energy configurations [18].

The kinematic phase space used in the analysis is $Q^{2}>$ $10 \mathrm{GeV}^{2}$ (DIS region; lower values can be considered as well) and $0.01<y<0.95$ (standard limits for event reconstruction using the electron method). Tagged DIS and nucleon structure extraction are simulated in the range $10^{-2} \lesssim x \lesssim$ $10^{-1}$. The main physical interest is in the measurements at

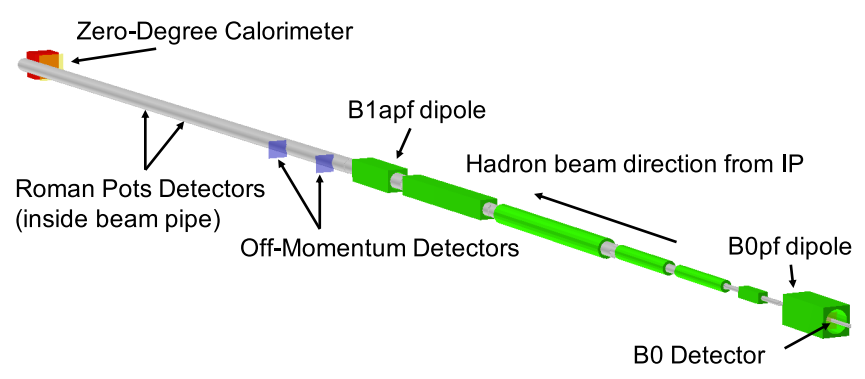

FIG. 4. The layout of the EIC far-forward area showing the four detector subsystems. A few of the relevant beamline magnets are also labeled for reference. The rectangular boxes are dipole magnets, and the cylinders are focusing quadrupoles. A schematic beam pipe is included in the drawing. The interaction point (IP) is at the bottom right, and the hadron beam direction is noted in the figure.

$x \gtrsim 0.1$, where the neutron and proton structure functions are significantly different, and where the free nucleon structure extraction with tagging provides a baseline for studies of nuclear modifications (EMC effect, antishadowing). Because the nucleon DIS process and the deuteron breakup are described independently in BeAGLE, the simulations of forward spectator detection do not depend significantly on the choice of $x$ and $Q^{2}$. We therefore include in the simulations also events at $x \ll 0.1$, where the statistical sample is large, but use them only for studying the detector performance.

In the analysis, $10^{8}$ (100 million) electron-deuteron DIS events were generated above $Q^{2}>10 \mathrm{GeV}^{2}$, corresponding to an integrated luminosity of $1 \mathrm{fb}^{-1}$ for electron-nucleon (proton or neutron) collisions. Of these, approximately half are events with an active proton, and half with an active neutron. The integrated luminosity represents a baseline number for DIS studies at EIC [19]. In the kinematic region considered here the uncertainties of the measured DIS cross section and extracted nucleon structure functions are dominated by systematic effects; the large event sample was chosen only to enable accurate phase space integration in the study of systematic effects.

\section{EIC far-forward detectors}

In the present study we perform full detector simulations using the subsystems specified in the EIC reference detector design, with the far-forward detector configuration presented in the EIC Yellow Report [19] and implemented in the EICRoOT framework [49]. EICROOT makes use of the ROOT Virtual Monte Carlo structure and GEANT4 [50] for detector simulations and contains classes for performing tracking and reconstruction tasks. A three-dimensional rendering of the layout and the subsystems is shown in Fig. 4.

The far-forward detector subsystems are optimized to make best use of the available space for detectors and maximize the geometric acceptance. The geometric acceptance for farforward nucleons (protons or neutrons) is a function of two variables: the polar angle of the outgoing nucleon at the interaction point relative to the ion beam axis, and the fractional longitudinal momentum of the outgoing nucleon relative to 
TABLE I. Summary of the geometric acceptance for far-forward protons and neutrons in polar angle $\theta$ and longitudinal momentum fraction $\zeta$, Eqs. (57) and (58), provided by the baseline EIC farforward detector design [19].

\begin{tabular}{lcrc}
\hline \hline Detector & Used for & $\theta$ accep. (mrad) & $\zeta$ accep. \\
\hline B0 tracker & $p$ & $5.5-20.0$ & N/A \\
Off-momentum & $p$ & $0.0-5.0$ & $0.45-0.65$ \\
Roman pots & $p$ & $0.0-5.0$ & $0.6-0.95^{\mathrm{a}}$ \\
Zero-degree calorim. & $n$ & $0.0-4.0$ & N/A \\
\hline \hline
\end{tabular}

${ }^{\text {aRoman pots acceptance at high values of } \zeta \text { depends on the optics }}$ choice for the machine.

the deuteron beam momentum [see Eq. (1)],

$$
\begin{aligned}
\theta_{p} & \equiv \frac{p_{p}(\text { transv })}{p_{p}(\text { longit })}, \\
\zeta_{p} & \left.\equiv \frac{p_{p}(\text { longit })}{p_{d}} \quad \text { (same for } \mathrm{n}\right) .
\end{aligned}
$$

Here $p_{d}$ is the total deuteron momentum (not the momentum per nucleon), so that a proton with the nominal longitudinal momentum $p_{p}$ (longit) $=p_{d} / 2$ has $\zeta_{p}=1 / 2$. Protons produced in the collision travel through the magnetic fields of the beamline magnets and experience bending in the dipoles inversely proportional to their longitudinal momentum. The proton acceptance therefore depends on both $\zeta_{p}$ and $\theta_{p}$. Note that protons from deuteron breakup have a magnetic rigidity $\approx 1 / 2$ of that of the deuteron beam and experience different bending; this effect is taken into account in the acceptance simulations; it is the main reason why the off-momentum detectors have been added to the far-forward region (see below). Neutrons are not affected by the magnetic fields and propagate from the interaction point on straight trajectories, with acceptance only limited by the magnet apertures. Therefore, the neutron acceptance does not depend on $\zeta_{n}$ and is only a function of $\theta_{n}$. Table I summarizes the geometric acceptance for far-forward protons and neutrons achieved with the present design [19]. We note that, in the $\theta$ and $\zeta$ range considered in the present study, the acceptance does not significantly depend on the azimuthal angle of the produced nucleon around the ion beam direction, and we assume it to be uniform in the azimuthal angle (see Appendix B).

For most of the DIS kinematics considered in the present study, the virtual photon direction is close to the ion beam direction, so that the nucleon longitudinal and transverse momenta relative to the ion beam axis approximately coincide with those in the collinear frame, and one can infer the $\zeta$ and $\theta$ values directly from the collinear frame variables (see Sec. II A)

$$
\left.\theta_{p} \approx 2 p_{p T} / p_{d}, \quad \zeta_{p} \approx \alpha_{p} / 2 \quad \text { (same for } \mathrm{n}\right) .
$$

The tagged measurements for nucleon structure extraction use spectator detection at rest-frame momenta $p_{p, n} \lesssim 100 \mathrm{MeV}$, corresponding to $0.9 \lesssim \alpha_{p, n} \lesssim 1.1$ and $p_{p T, n T} \lesssim 100 \mathrm{MeV}$. With the beam momentum $p_{d} / 2=110 \mathrm{GeV}$ this implies forward detection in the range

$$
\theta_{p, n} \lesssim 1 \mathrm{mrad}, \quad 0.45 \lesssim \zeta_{p, n} \lesssim 0.55
$$

Note that the same measurements at a lower beam energy would cover a proportionally wider range in $\theta$ and $\zeta-\frac{1}{2}$.

In the following we summarize the main features of the subsystems as relevant to the present study, in the order in which they appear when moving away from the interaction point; see Fig. 4. Details can be found in Refs. [18,19].

BO spectrometer. The B0 spectrometer consists of four layers of silicon tracking planes embedded in the first dipole magnet after the interaction point (B0pf). This subsystem is designed for reconstructing charged particles with angles $5.5<\theta<20.0 \mathrm{mrad}$, such as large-angle protons from nuclear breakup. It is not used in the present study.

Off-momentum detectors. The off-momentum detectors are designed to optimally tag charged particles with a magnetic rigidity $\approx 1 / 2$ that of the beam. The present design achieves an angular acceptance $0.0<\theta<5.0 \mathrm{mrad}$, similar to that of the Roman pot detectors tagging particles with rigidity $\approx 1$ (see below). In the Yellow Report [19] and the present study, the off-momentum detectors were placed just after the Blapf dipole magnet; the final design of the beam pipe and vacuum system may require them to be placed elsewhere. The off-momentum detectors are the subsystem mainly used for proton tagging in the present study. The $p_{p T}$ resolution is $\approx 20 \%$ at $p_{p T}=100 \mathrm{MeV}$, with the smearing mainly resulting from the transfer matrix used for reconstruction. The off-momentum detectors have in general worse overall resolution than the Roman pots because the transfer matrix for off-momentum particles requires a more sophisticated implementation than what was available at the time of this study.

Roman pots. The Roman pots detector is situated $\approx 27$ meters downstream from the interaction point and consists of silicon sensors placed in Roman pot vessels or in shields without pots. They are injected into the beamline vacuum a few millimeters from the hadron beam. The Roman pots subsystem is used for capturing charged particles with small scattering angles $0.0<\theta<5.0 \mathrm{mrad}$ and rigidities similar to that of the beam; the acceptance for protons from deuteron breakup is $\zeta_{p} \gtrsim 0.6$. In the present study of low-momentum tagging, the spectator protons rarely impinge on the Roman pots; the majority are captured by the off-momentum detectors. However, the Roman pots become important in tagging experiments with $\alpha_{p} \gtrsim 1.2$, as are used in studies of nuclear modifications or short-range correlations in the deuteron. For the Roman pots detector, the $p_{p T}$ resolution is $\approx 10 \%$ at $p_{p T}=$ $100 \mathrm{MeV}$, with the smearing being driven primarily by the beam angular divergence.

Zero-degree calorimeter. Because neutrons are not affected by the magnetic field, the geometric acceptance is determined by the apertures of the various beam elements that the far-forward neutrons have to traverse before detection. The present EIC interaction region design promises far-forward neutron acceptance at angles $\theta<4.0 \mathrm{mrad}$, which allows for tagging neutrons in a variety of final states of interest for physics studies, including the present one. Detection of the outgoing neutrons requires hadronic calorimetry far enough downstream from the interaction point to allow the neutrons to exit the beam pipe and impinge on the detector. This is achieved with a zero-degree calorimeter, which will also have an electromagnetic calorimeter for tagging photons, and a 
layer of silicon for vetoing charged particles. In the present study we assume a hadronic calorimeter with the same performance as in the Yellow Report [19],

$$
\frac{\Delta E}{E}=\frac{50 \%}{\sqrt{E}} \oplus 5 \%, \quad \frac{\Delta \theta}{\theta}=\frac{3 \mathrm{mrad}}{\sqrt{E}} .
$$

\section{Momentum reconstruction}

The measurement of the momentum of the spectator nucleon (proton or neutron) is essential for the analysis of tagged DIS and a main concern of the present study. The reconstruction of the far-forward nucleon momentum is impacted by several detector and beam effects [19]. First, there are effects intrinsic to the detectors themselves, e.g., finite pixel sizes or hadronic calorimeter energy resolution. Second, the momentum reconstruction algorithm for the Roman pots and off-momentum detectors depends on a transport matrix, which connects the spatial coordinates of the particles detected in the relevant far-forward detectors with their momenta at the interaction point. Currently this transport matrix is implemented as a linear transformation, which works very well for the Roman pots system where trajectories are transported from the interaction point to the detector linearly, but does not accurately describe particles with momenta very different from the nominal beam momentum, as seen in the off-momentum detectors. The strategy employed in this study was to calculate the matrix for the off-momentum detectors for proton trajectories where $\zeta \approx 0.5$, allowing for minimal reconstruction smearing in the region of interest for the present study of pole extrapolation. Third, there are beam-related effects, such as the beam angular divergence, the beam momentum spread, and the vertex smearing induced by the crab cavities used to prevent a luminosity drop in bunch collisions at the EIC crossing angle of $25 \mathrm{mrad}$. All these effects are included in the simulations in the present study, to assess the impact on the physics measurements.

The detector and beam effects on the momentum reconstruction are quantified by processing the BeAGLE events with the GEANT implementation of the far-forward detector systems. Because of the large size of the event sample required for the present physics study (see Sec. III B), running the entire generated BeAGLE sample through the GEANT full simulations proved impractical. Instead, a representative subsample was processed through the full simulations to generate resolution functions for the reconstruction of momentum and energy for both protons and neutrons. These distributions were then read as input into the analysis code and used to smear the energy and individual momentum components as the various quantities were calculated, to inject the effects of detector reconstruction into the analysis. For reference, the distributions are presented in Appendix B; they can be used in other physics studies requiring far-forward proton or neutron detection.

\section{ANALYSIS AND RESULTS}

\section{A. Deuteron cross section measurement}

We now present the simulated analysis of tagged DIS and nucleon structure extraction and the lessons learned and
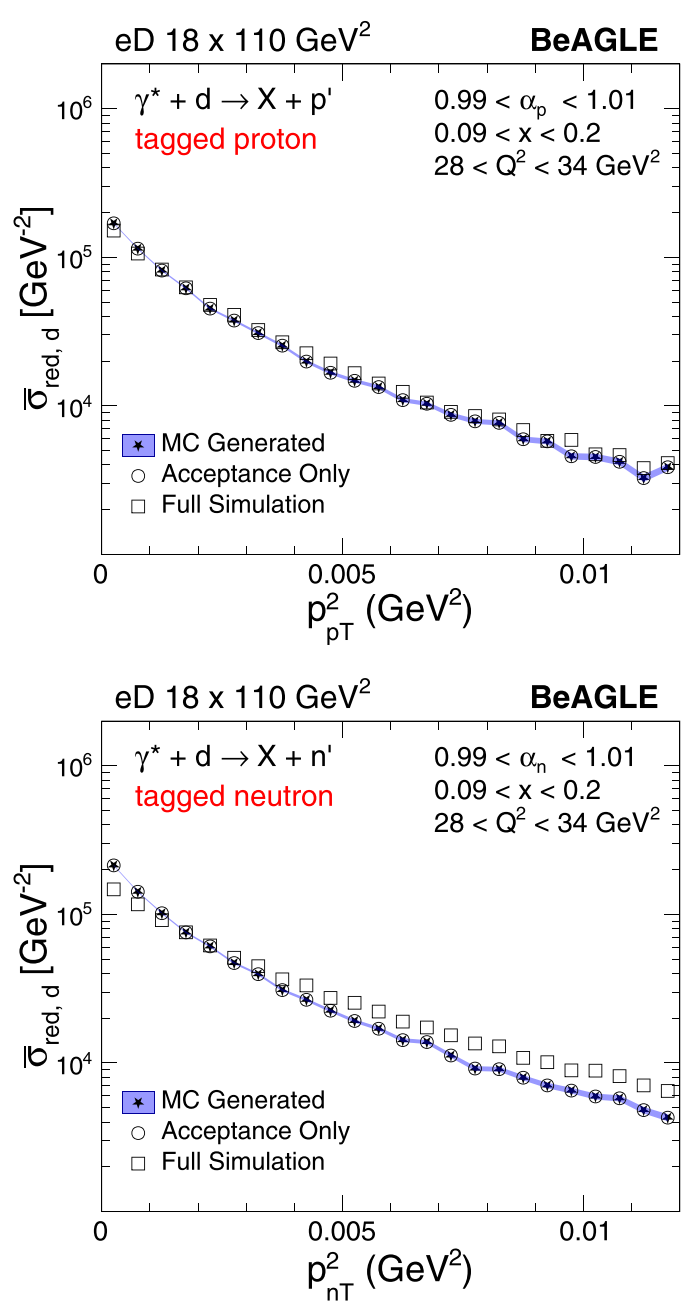

FIG. 5. The reduced cross sections of deuteron DIS with proton (top) and neutron tagging (bottom), Eq. (49), as functions of $p_{p T}^{2}$ and $p_{n T}^{2}$, as extracted from simulated measurements at the EIC. The reduced cross sections plotted here have dimensions of $\mathrm{GeV}^{-2}$ because they are differential in the tagged nucleon momentum; see Eq. (14). Stars and bands: Truth distributions from BeAGLE. Circles: Distributions reconstructed with detector acceptance only. Squares: Distributions reconstructed with full simulations.

results obtained from the study. The analysis follows the steps described in Sec. IIF and uses the method of pole extrapolation. The material is the BeAGLE event sample for electron-deuteron DIS of Sec. III B, consisting of tagged proton and neutron events; the simulated analysis applies the detector acceptance and the smearing distributions representing the detector and beam effects on the spectator nucleon momentum reconstruction of Sec. III D. In each step we consider both proton and neutron tagging and compare the two channels.

In the first step, we measure the tagged DIS cross section and extract the reduced cross section by removing the flux factor, as specified in Eqs. (48) and (49) for proton tagging and the corresponding formulas for neutron tagging. Figure 5 shows the extracted $\phi_{p}\left(\phi_{n}\right)$-averaged reduced cross sections $\bar{\sigma}_{\text {red, } d}$, as functions of the spectator transverse momentum $p_{p T}^{2}$ $\left(p_{n T}^{2}\right)$. The plots show the generator-level distributions based 
on the BeAGLE events, the distributions reconstructed with acceptance effects only, and the distributions reconstructed with the full simulations. The example covers the kinematic range is $28<Q^{2}<34 \mathrm{GeV}^{2}, 0.09<x<0.2$, and $0.99<$ $\alpha_{p}\left(\alpha_{n}\right)<1.01$; similar results are obtained in other ranges. Comparing the truth and acceptance-only results in Fig. 5, one sees that the acceptances for both proton and neutron spectators are close to $100 \%$ in the transverse momentum range covered here. Comparing the acceptance-only and the full simulations, one sees the impact of the detector and beam smearing effects on the reconstruction, typically a few percent for proton tagging and up to $\approx 30 \%$ for neutron tagging. In the case of neutron detection, the zero-degree calorimeter energy resolution is the dominant source of momentum smearing.

\section{B. Implementation of pole removal}

In the second step of the analysis, we divide the deuteron reduced cross section by the pole factor of the deuteron spectral function to extract the ratio (50), which gives access to the nucleon reduced cross section. This "pole removal" is the most critical step of the experimental analysis and requires careful study. The pole factor in Eq. (50) is a theoretical function that needs to be evaluated at the experimentally reconstructed spectator momentum. Because of the steep momentum dependence of the reduced cross section and the pole factor, the uncertainties in the spectator momentum reconstruction can have a large numerical effect on the result. There are two possible approaches to implementing the pole removal in the experimental analysis: (i) compute the ratio (50) on an event-by-event basis, i.e., evaluate the pole factor at the actual momentum of the event; (ii) compute the ratio on an eventaveraged basis, i.e., evaluate the pole factor at an average momentum in a finite bin. Both have apparent advantages and disadvantages. The event-by-event approach is theoretically more accurate because of the steep momentum dependence of the functions; however, in the experimental analysis the reconstructed momenta are subject to large uncertainties due to detector and beam effects. The event-averaged approach can be corrected statistically for detector and beam effects; however, it retains uncertainties from the finite bin size. The tradeoffs between these effects are generally different for proton and neutron tagging can be explored in our simulations.

We have performed a detailed study of the two approaches to pole removal for both proton and neutron tagging. Figure 6 compares the results of the two approaches in a typical $x, Q^{2}$, and $\alpha$ bin. The plots show the ratio (50) extracted with the event-by-event and average approaches, first in an analysis using the original MC events (exact momenta), and second in an analysis with full simulations (momentum smearing from detector and beam effects). In the case of proton tagging (upper plot), one sees that the event-by-event and average results are in good agreement when the analysis is performed with MC events, showing the theoretical consistency of the two approaches. The event-by-event and average results are also in reasonable agreement when the analysis is performed with the full simulations, showing that the overall impact of the proton momentum smearing is moderate. In the case of neutron tagging (lower plot), the situation is very different. One observes
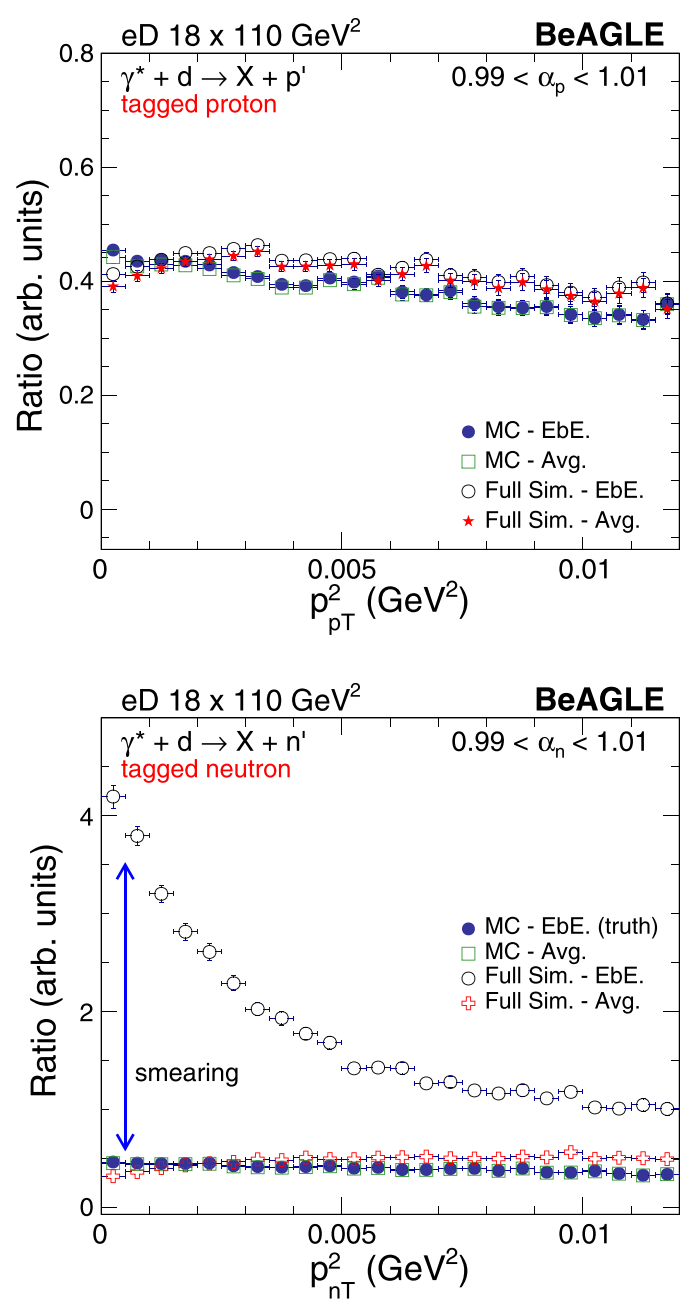

FIG. 6. Comparison of the event-by-event (EbE) and eventaveraged (Avg) approaches to pole removal in proton (top panel) and neutron tagging (bottom panel). The plots show the ratio of the deuteron cross section and the pole factor, Eq. (50), extracted in different ways: Solid circles: MC events (exact momenta), event-by-event approach. Open squares: MC events, event-averaged approach. Open circles: Full simulations (reconstructed momenta with smearing), event-by-event approach. Crosses: Full simulations, event-averaged approach. The results shown in the plots correspond to a typical $\left(x, Q^{2}\right)$ bin.

that that the event-by-event and average results are again in good agreement when the analysis is performed with MC events, as expected. However, when the the analysis if performed with full simulations, the results of the event-by-event approach differ qualitatively from those of the event-averaged approach. The differences are caused by the substantial smearing of the reconstructed neutron momentum variables $\alpha_{n}$ and $p_{n T}$, which has a large numerical effect on the calculated pole factor. In particular, the smearing of $\alpha_{n}$ [caused mainly by the zero-degree calorimeter energy resolution, Eq. (61)] has a major numerical effect in the evaluation of the pole position $a_{T}^{2}\left(\alpha_{n}\right)$ [given by Eq. (41) with $\alpha_{p} \rightarrow \alpha_{n}$ ] and causes $O$ (1) differences in the calculated pole factor. In the eventby-event approach these smearing effects cannot be corrected, and the results are fully exposed to the detector and beam 


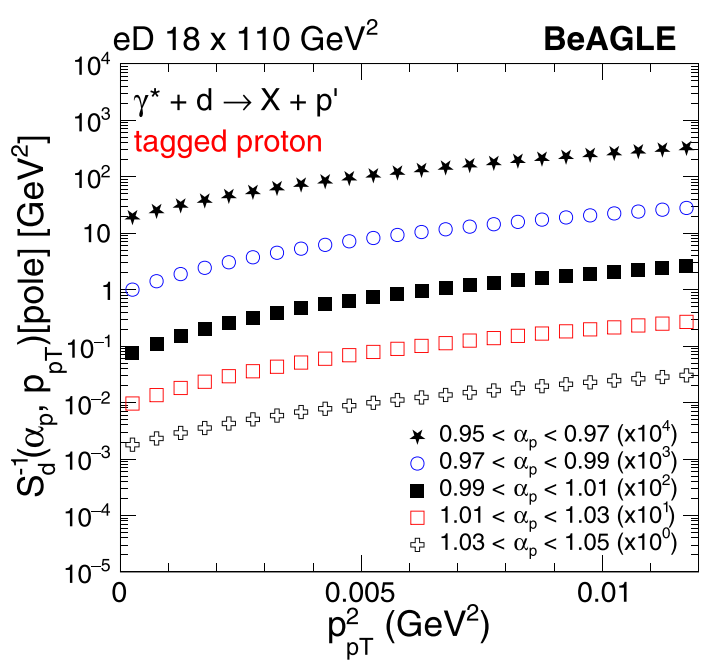

FIG. 7. The inverse pole factor in proton tagging, $1 / \mathcal{S}_{d}\left(\alpha_{p}, p_{p T}\right)$, Eq. (44), as a function of $p_{p T}^{2}$, for various values of $\alpha_{p}$. The function was reconstructed from full simulations using the event-averaged approach. The results for different $\alpha_{p}$ are offset by powers of 10 for visibility.

uncertainties. In the event-averaged method the smearing effects can be corrected, and the results are in reasonable agreement with those obtained from MC events without smearing. Our study shows that for neutron tagging the event-averaged approach is the only realistic method for performing the pole removal. For proton tagging an event-byevent approach might be considered; however, its performance depends on the actual detector resolutions, and a final assessment is not possible at this stage. Overall, the event-averaged approach to pole removal is more realistic and more robust against detector effects, and we adopt it in the present analysis.

Figure 7 shows the reconstructed pole factor in proton tagging using the event-averaged approach. The plot shows $1 / \mathcal{S}_{d}$ as a function of $p_{p T}^{2}$ in several bins of $\alpha_{p}$; this is the function that the deuteron cross section in Fig. 5 is multiplied with in order to extract the neutron cross section. One sees that the experimentally reconstructed pole factor is a smooth function and follows the theoretical function shown in Fig. 3.

\section{Nucleon structure from pole extrapolation}

In the third step of the analysis, we extrapolate the deuteron cross section after pole removal to the nucleon pole $p_{p T}^{2}\left(p_{n T}^{2}\right) \rightarrow-a_{T}^{2}$, where it gives the free nucleon cross section; see Eq. (52). Figure 8 shows the simulated data and the extrapolation procedure for both proton and neutron tagging. The bands show the $p_{p T}^{2}\left(p_{n T}^{2}\right)$ dependence of the cross section after pole removal, Eq. (50), as obtained from the MC data with acceptance effects only (no smearing). One sees that the dependence of this quantity on $p_{T}^{2}$ is very weak, because most of the $p_{T}^{2}$ dependence of the tagged cross section has been removed by the pole factor (see also Fig. 3), and that the data indicate a regular distribution around a smooth curve. The extrapolation to negative $p_{T}^{2}$ can therefore be performed with a low-order polynomial fit. The degree of the fitting polynomial and the choice of $p_{T}^{2}$ range for the fit are a matter of opti-
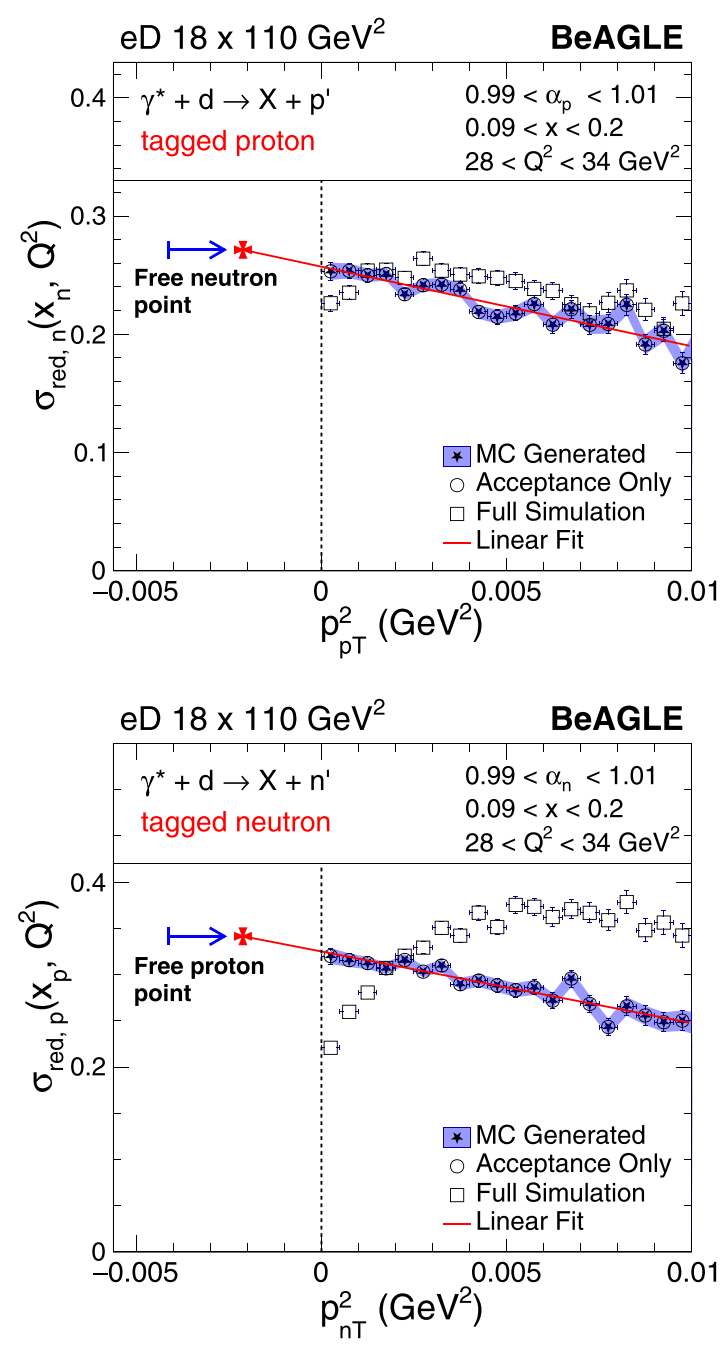

FIG. 8. Pole extrapolation and free nucleon reduced cross section extraction in spectator tagging. Top: Neutron reduced cross section with proton tagging. Bottom: Proton reduced cross section with neutron tagging. The data show the deuteron reduced cross sections divided by the pole factor, Eq. (52), as functions of $p_{p T}^{2}\left(p_{n T}^{2}\right)$; these quantities are dimensionless and coincide with the nucleon reduced cross sections when extrapolated to the pole. Stars and bands: MC data (generator level). Circles: Reconstructed with acceptance only. Squares: Full simulations including acceptance and smearing effects (these data show the raw smearing effects and have not been corrected). The lines shows the first-degree polynomial fits used for the pole extrapolation. The fit functions are evaluated at the pole position Eq. (41), where they give the free nucleon reduced cross sections (denoted by the arrows).

mization and determine the fit uncertainty (see Sec. V); the example in the figure is representative and shows a first-order fit over the range $0<p_{T}^{2}<(100 \mathrm{MeV})^{2}$. The free nucleon reduced cross section and its uncertainty are obtained by evaluating the fit at the pole momentum $p_{p T}^{2}\left(p_{n T}^{2}\right)=-a_{T}^{2}$. Note that the extrapolation relies essentially on the EIC far-forward acceptance extending down to $p_{T}^{2}=0$ for both protons and neutrons; any acceptance limit $p_{T}^{2}>0$ would increase the extrapolation distance and uncertainty. 

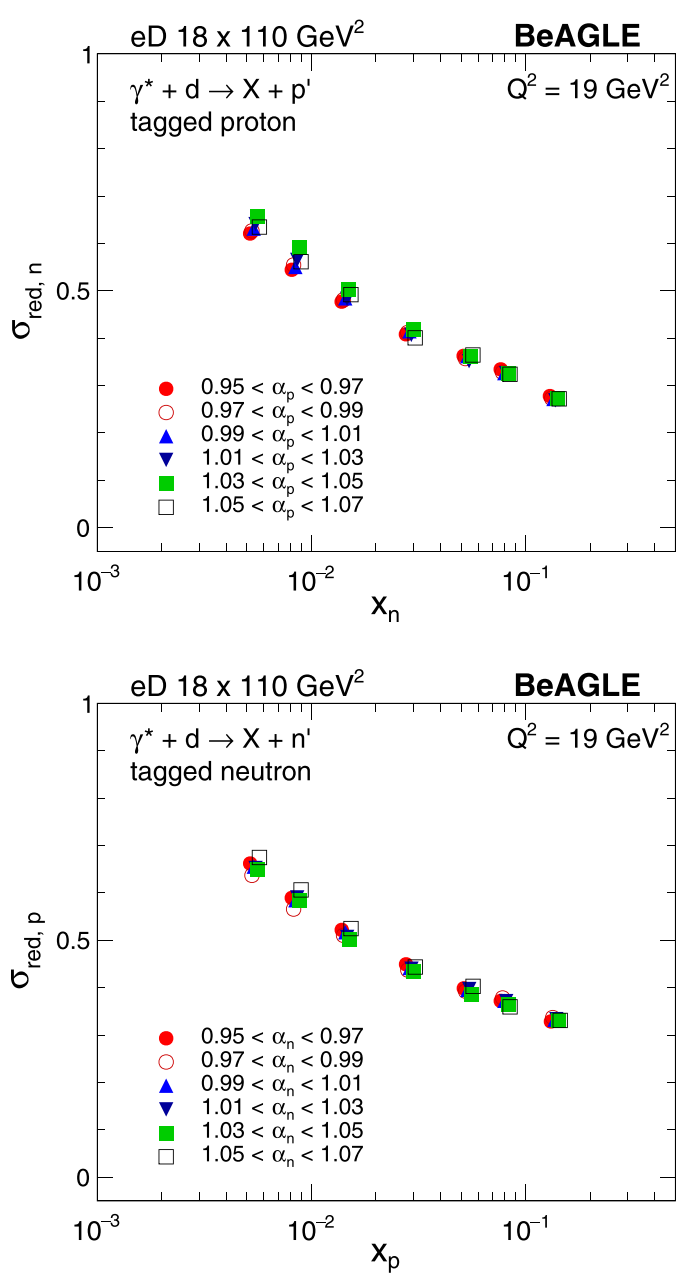

FIG. 9. The free neutron (top) and proton (bottom) reduced cross sections extracted with pole extrapolation, as functions of $x_{n}$ and $x_{p}$, respectively. The plots show the results of extractions performed at different $\alpha_{p}\left(\alpha_{n}\right)$. The nucleon reduced cross sections plotted here are dimensionless.

In Figure 8 the extrapolation is performed with the MC data with acceptance effects only. The plots also show the distributions obtained from the full simulations, which include the effects of momentum smearing in the cross section and the pole factor. One sees that these distributions differ from the generator-level distributions by $\approx 10 \%$ in the case of proton tagging, and $\approx 30 \%$ in neutron tagging. In an actual experiment the smearing effects will be corrected by an unfolding procedure, which is expected to eliminate most of the differences. Performing the extrapolation with the original MC distributions therefore presents a realistic picture of nucleon structure extraction in the actual experiment.

Figure 9 shows the free neutron and proton reduced cross sections measured via pole extrapolation, Eq. (52), at several values of $\alpha_{p}$ and $\alpha_{n}$. The reduced cross sections are presented as functions of $x_{n}$ and $x_{p}$, Eqs. (28) and (34), the nucleonlevel scaling variables whose values are fixed by the spectator kinematics. The results shown here have been corrected for artifacts resulting from the treatment of the electron-nucleon subprocess kinematics in BeAGLE, by applying the factor
(54) (see Sec. III A; this correction will not be needed in a real experiment). An important feature of tagging is that the same value of $x_{n}\left(x_{p}\right)$ can be realized with different combinations of $x$ and $\alpha_{p}\left(\alpha_{n}\right)$, allowing one to measure the same physical nucleon cross section in different settings of the external DIS and spectator kinematics. Figure 9 shows that the results obtained at different values of $\alpha_{p}\left(\alpha_{n}\right)$ agree at the level of 5-10\%; the small differences result from the event-averaged pole-removal procedure and could be reduced by corrections (see Sec. IIF). This provides a crucial test of the simulations and the robustness of the extraction procedure. Note that in extractions at $\alpha \neq 1$ the pole extrapolation has to cover a larger distance than at $\alpha=1$, because the nucleon pole position (41) increases quadratically in $(\alpha-1)$.

\section{Validation of nucleon structure extraction}

The analysis simulated here extracts the reduced cross section of the free nucleon from the tagged DIS data using the method of pole extrapolation. We can validate the results by comparing the extracted nucleon cross section with the input at the generator level. In the present study using BeAGLE, this validation can be accomplished simply by comparing the result of the pole extrapolation ("Method II" of Sec. IID) with the nucleon cross section obtained by integrating over the spectator momentum ("Method I" of Sec. II D). [As explained in Sec. IIE, this is possible because BeAGLE implements the impulse approximation without dynamical initial-state modifications or final-state interactions, so that the integration over the spectator momentum recovers the nucleon cross section; see Eq. (39).] While specific to this generator, this comparison offers a very convenient way to test the result of the pole extrapolation.

Figure 10 shows the comparison of the nucleon reduced cross section computed using integration over the spectator momentum in BeAGLE (Method I) and the result of the pole extrapolation (Method II). The results of the two methods agree within $\approx 2 \%$ in the $\left(x, Q^{2}\right)$ range covered in our study. This level of agreement is consistent with the accuracy of the event-averaged pole removal approach as presently implemented (see Sec. IV B); the accuracy could be improved by applying bin-centering corrections. For an even more stringent test, Fig. 11 shows the the same comparison using the pole extrapolation result obtained with the event-by-event approach, which is free of the bin centering corrections (see Sec. IV B). Now the agreement between the integration and the pole extrapolation methods is at the level $\lesssim 1 \%$, which is the expected accuracy of the pole extrapolation. This shows that the small discrepancies observed in Fig. 10 are indeed due to the accuracy of the event-averaged pole removal approach. Altogether, these tests show agreement between the integration and the pole extrapolation methods at the expected level of accuracy and validate the results of the pole extrapolation.

\section{DISCUSSION}

\section{A. Experimental uncertainties and effects}

We now discuss the experimental and theoretical uncertainties arising in the proposed tagged DIS measurements with 

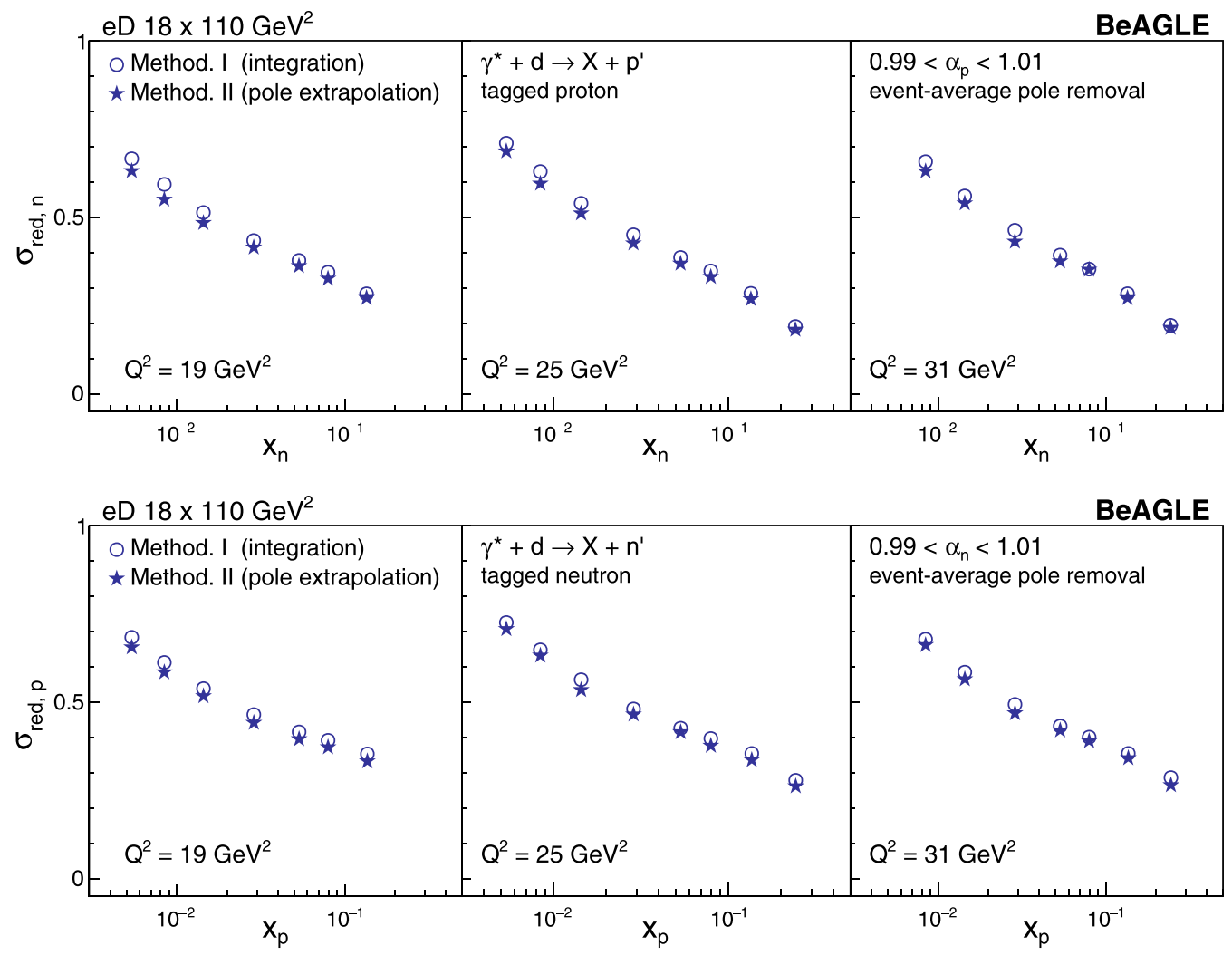

FIG. 10. Validation of nucleon structure extraction with spectator tagging in BeAGLE. The plots show the neutron (proton) reduced cross sections $\sigma_{\text {red }, n}\left(\sigma_{\text {red, } p}\right)$ as functions of $x_{n}\left(x_{p}\right)$, extracted with two different methods (see Sec. II D). The nucleon reduced cross sections plotted here are dimensionless. Stars: Integration over spectator momentum (Method I). Circles: Pole extrapolation in spectator momentum (Method II). Here the event-averaged approach was used in removing the pole factor (see Sec. IIF).

EIC and the nucleon structure extraction with pole extrapolation. Because our study refers to simulated measurements with a future facility, the analysis of uncertainties is necessarily different from that of actual measurements with an existing facility. Some of the experimental effects included in the simulations of Sec. IV cannot be fully quantified because they depend on the final detector performance, while other effects cannot even be included because a design of the necessary components is not available. In the following discussion we therefore address both the status of the modeling of the various effects and their impact on the analysis. Our goal is to provide an assessment of the uncertainties that is realistic and can be extended and improved with future developments.

Statistical uncertainties. Tagged DIS has the same rates as inclusive DIS on the deuteron, only differentiated in the spectator nucleon momentum. In the present study of free nucleon structure extraction we use spectator momenta $p_{p T}\left(p_{n T}\right) \lesssim$ $100 \mathrm{MeV}$, which correspond to average nuclear configurations and account for the bulk of the deuteron momentum distribution. The integrated luminosity of $1 \mathrm{fb}^{-1}\left(\approx 10^{8}\right.$ events) is more than sufficient for the differential measurements of the $p_{T}^{2}$ distributions in the $\left(x, Q^{2}\right)$ region considered here. The nucleon structure extraction is not limited by statistics and the resulting overall uncertainties are dominated by systematic effects. The situation will be different in future studies of nuclear modifications, which access both larger $x \gtrsim 0.3$ and $p_{p T}\left(p_{n T}\right) \approx 300-600 \mathrm{MeV}$, where the rates are much lower.
DIS variable reconstruction. The DIS variables $x$ and $Q^{2}$ in tagged DIS are reconstructed in the same way as in standard inclusive DIS. The uncertainties associated with the reconstruction have been studied extensively in inclusive DIS simulations and are described in the Yellow Report [19]. The DIS kinematics covered in the present study is nonexceptional, and the performance of the standard electron method is expected to be at the percent level.

Spectator momentum reconstruction. The reconstruction of the far-forward proton and neutron momenta is affected by various detector and beam effects. The present simulations include the following effects: (i) intrinsic detector smearing (both protons and neutrons); (ii) Deuteron beam angular divergence; (iii) deuteron beam momentum spread; and (iv) crab cavity rotations. These effects have been evaluated with the current EIC accelerator and detector design, and their aggregate effect on the signal (before correction) is shown in the "Full Simulation" results in Figs. 5, 6, and 8. The contributions of the individual effects can be seen in the summary plots in Appendix B. Note that the impact of the various effects is different for protons and neutrons: the dominant effect for protons comes from the angular divergence of the deuteron beam (ii), while the neutrons suffer mostly from the energy resolution of the zero-degree calorimeter (i).

Several other effects can influence the far-forward nucleon detection but have not yet been included in the simulations: (v) beam pipe design and (vi) nonlinear transport matrix. These 

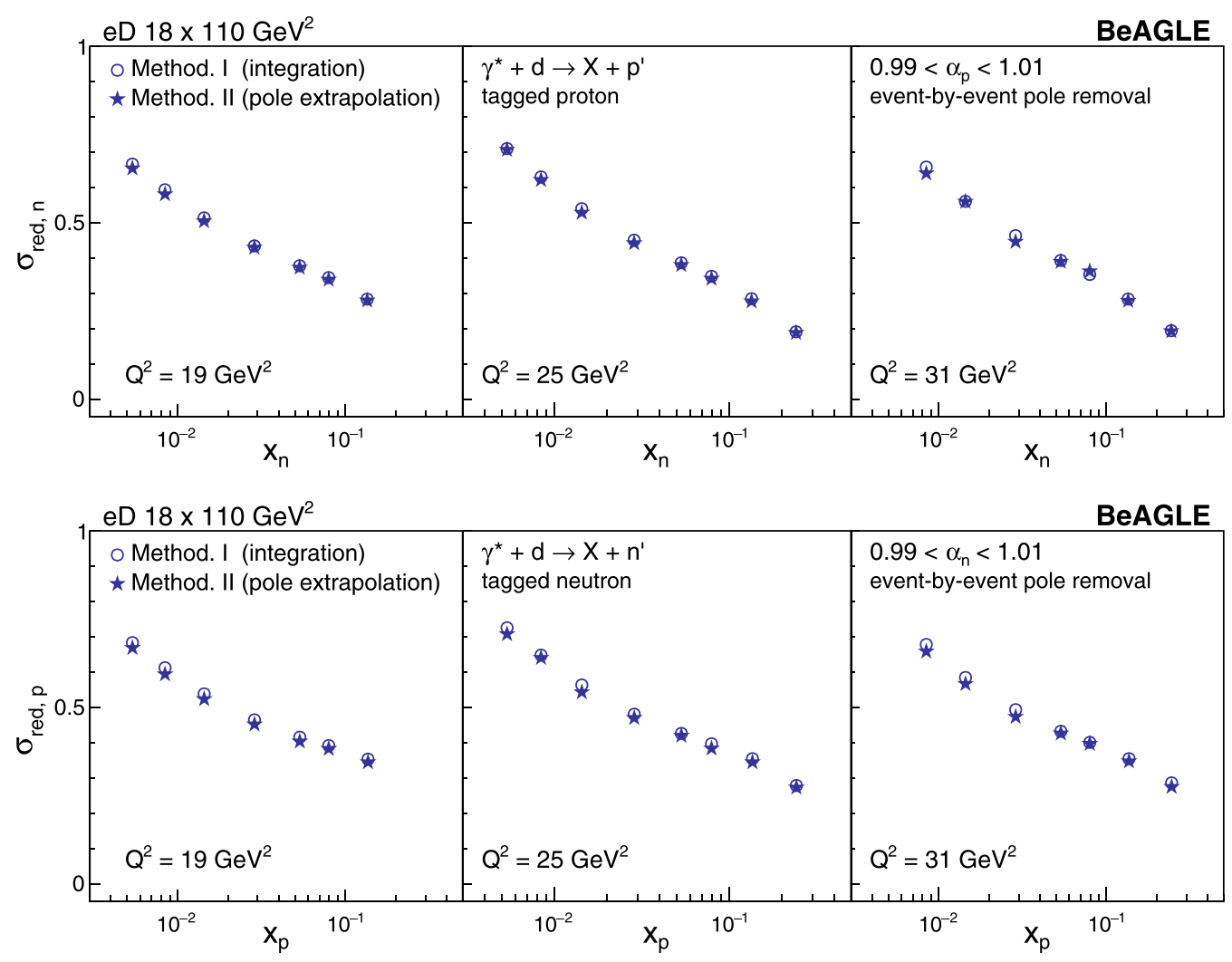

FIG. 11. Validation of nucleon structure extraction with spectator tagging in BeAGLE (see also Fig. 10). The plots show the neutron (proton) reduced cross sections $\sigma_{\text {red, } n}\left(\sigma_{\text {red, } p}\right)$ as functions of $x_{n}\left(x_{p}\right)$, extracted with two different methods (see Sec. II D). The nucleon reduced cross sections plotted here are dimensionless. Stars: Integration over spectator momentum (Method I). Circles: Pole extrapolation in spectator momentum (Method II). In contrast to Fig. 10, here the event-by-event approach was used in removing the pole factor (see Sec. II F).

effects can be included as the technical design or specification of these elements becomes available. The beam pipe design (v) will mostly impact the overall detection efficiency; the nonlinear transport matrix (vi) will affect the assessment of the momentum smearing for protons in the off-momentum detectors. These effects are not expected to substantially modify the findings of the present simulations.

In the actual experimental analysis the beam and detector effects described here will be corrected through an unfolding procedure. The systematic uncertainty in the final physics results is not given by the size of the original effects, but by the accuracy with which they can be corrected. The unfolding procedure will use apparatus information (design, performance) that is not available at present. Progress in detector technology and correction algorithms in the time until the EIC experiments are performed will significantly improve the estimated accuracy of the correction procedure. For these reasons we presently cannot perform a quantitative assessment of the systematic uncertainties after corrections. The important result of our study is that the aggregate effects before corrections are $\approx 10 \%$ for protons and $\approx 30 \%$ for neutrons; see Fig. 10 . A reasonable unfolding procedure is expected to be able to correct these effects with a final accuracy at the percent level, which will be sufficient for an impactful physics analysis.

Backgrounds. In DIS measurements there are different sources of backgrounds such as beam-gas interactions, particles coming from hadronic final states, photoproduction, etc. These backgrounds and not studied here, as they are common to all inclusive DIS measurements and not specific to spectator tagging [19]. Forward nucleons produced through target fragmentation represent a theoretical background and are discussed below.

\section{B. Theoretical and fit uncertainties}

Uncertainties in pole extrapolation. The extraction of the free nucleon structure function through pole extrapolation is subject to specific uncertainties; see Sec. IV C. The first uncertainty is related to the effects in the event-averaged pole removal approach described in Sec. IIF, which can be corrected statistically. The error of this correction remains as a source of uncertainty and is estimated to be $\approx 1-2 \%$. The second uncertainty is associated with the polynomial fits used to perform the extrapolation. The degree of the polynomial and the fitting range are determined in an optimization process that takes into account the average variation of the function and the fluctuations of the data; the optimal configuration is approximately stable against variation of the degree and range. We have performed fits using polynomials from first to fifth order, each with different fit ranges. Combining all the fit results and comparing to the truth value, the best fit is found to be the first- or second-order polynomial with a fit range of $0<p_{p T}^{2}\left(p_{n T}^{2}\right)<0.01 \mathrm{GeV}^{2}$. By comparing to other fit configurations, the final extrapolation uncertainty is estimated 
at $\approx 1-2 \%$ for the fits at $\alpha_{p}\left(\alpha_{n}\right) \approx 1$. We emphasize that the optimization of the fit and the estimate of the fit uncertainty ultimately depend on the quality of the data after pole removal and will have to be revisited when the unfolding procedure can be modeled or when actual data become available.

The extrapolation uncertainty is also affected by the distance of the pole from the physical region; see Eq. (41). The distance is minimal for $\alpha_{p}=1$, where $a_{T}^{2}\left(\alpha_{p}\right)=a^{2}=$ $0.0021 \mathrm{GeV}^{2}$, and increases quadratically for $\alpha_{p} \neq 1$ (same for $\left.\alpha_{n}\right)$. For the $\alpha_{p}$ values used in the free neutron structure extraction, $\alpha_{p} \approx 0.95-1.05$, the distances cover the range $a^{2} \leqslant$ $a_{T}^{2} \lesssim 2 a^{2}$. In this limited range we find that the extrapolation uncertainty does not strongly depend on the extrapolation distance (when the extrapolation is performed with a linear fit, the intercept and the slope contribute comparably to the extrapolation uncertainty), and the quoted uncertainty covers the entire range of $\alpha_{p}$ considered here.

Theoretical uncertainty of pole factor. The pole extrapolation method relies on the fact that the division by the pole term removes the singular $p_{p T}^{2}$ dependence of the deuteron spectral function and leaves only a smooth polynomial dependence in $p_{p T}^{2}+a_{T}^{2}$, normalized to unity at the pole; see Fig. 3 and Eq. (47) (same for $p_{n T}^{2}$ ). This follows from the analytic properties of the spectral function: at low $p_{p T}^{2}$ the function is dominated by the pole as the nearest singularity; the contributions of far-away singularities are smooth; and the pole dominance becomes exact as one approaches the pole. The analytic properties are model independent in the sense that they are based on general features of strong interactions (finite range of the $N N$ interaction, and universal behavior of the deuteron wave function at large distances) and common to all deuteron structure models considered here; see Appendix A 5. The position of the pole is fixed by the deuteron binding energy. The only model parameter in the pole of the spectral function is the residue $C$, Eq. (45), which is given in terms of the residue of the pole of the wave function, $\Gamma$, Eq. (A27). This parameter is determined by low-energy nuclear structure calculations and independent low-energy deuteron breakup measurements and known with an accuracy of $\lesssim 1 \%$; see Appendix A 5 and Table II. In an analysis with actual experimental data, the uncertainty in $C \propto \Gamma^{2}$ would cause an overall normalization uncertainty of the extracted cross section. In the present analysis with simulated events, the value of $\Gamma$ in the deuteron structure model of the BeAGLE generator is known; the same value is used in evaluating the pole factor Eq. (44) in the analysis; and the simulation and validation procedures do not include the uncertainty related to this parameter.

Deuteron structure in physical region. In the physical region $p_{p T}^{2}>0$ the tagged cross section is subject to model dependence from the deuteron spectral function. However, the analytic properties ensure that, after removal of the pole factor, these model uncertainties are proportional to $p_{p T}^{2}+a_{T}^{2}$ and vanish at the pole, and that they are numerically small in the $p_{p T}^{2}$ region used for extrapolation. As such, they can cause small changes of the slope of the $p_{p T}^{2}$ dependence of the data after pole removal (see Fig. 8), but do not change the approximately linear dependence or the result of the extrapolation. More precisely, the $p_{p T}^{2}$ dependence of the spectral function in the physical region is of the form

$$
\begin{aligned}
\mathcal{S}_{d}\left(\alpha_{p}, p_{p T}\right)= & \frac{C}{\left(p_{p T}^{2}+a_{T}^{2}\right)^{2}}\left\{1+b_{1}\left(p_{p T}^{2}+a_{T}^{2}\right)\right. \\
& \left.+O\left[\left(p_{p T}^{2}+a_{T}^{2}\right)^{2}\right]\right\} \\
& \times\left[C, a_{T}^{2}, b_{1} \equiv \operatorname{functions}\left(\alpha_{p}\right)\right] .
\end{aligned}
$$

The first factor is the model-independent free nucleon pole, Eq. (44); the expression in braces describes the deviations from the pole due to finite-range $N N$ interactions, in the form of an expansion in $\left(p_{p T}^{2}+a_{T}^{2}\right)$. In the region $p_{p T}^{2} \lesssim$ $0.01 \mathrm{GeV}^{2}$, the contribution of the first-order term $b_{1}\left(p_{p T}^{2}+\right.$ $a_{T}^{2}$ ) is $\lesssim 0.3$ (see Fig. 3). The model dependence from deuteron structure can be represented as a change of the coefficient of the linear term, $b_{1} \rightarrow b_{1}+\delta b_{1}$. The corresponding change in the tagged DIS cross section, after removal of the pole factor, is

$$
\frac{\delta \mathcal{S}_{d}}{\left.\mathcal{S}_{d} \text { [pole }\right]}=\delta b_{1}\left(p_{p T}^{2}+a_{T}^{2}\right) .
$$

This amounts to a change of the slope of the linear $p_{p T}^{2}$ dependence of the data in Fig. 8, with no change of the value at the pole position $p_{p T}^{2}=-a_{T}^{2}$. Based on a conservative estimate of the model uncertainty in the deuteron wave function at low momenta, we can assume $\delta b_{1} / b_{1} \lesssim 10 \%$. Altogether, we obtain that the effect of the model dependence would be a $10 \%$ change of the slope of data in Fig. 8, with no effect on the pole value. Such a change would amount to a small deterministic "resetting" of the data in the fit region by a linear function, which does not interfere with the polynomial fit or the result of the extrapolation; the change in the data simply would be compensated by a change of the linear term in the fit. We conclude that the model dependence of the deuteron spectral function in the physical region is contained in the fit uncertainty estimated above and does not constitute a separate source of uncertainty in the present context.

Modified nucleon structure. Similar arguments apply to modifications of the structure of the active nucleon due to nuclear binding. These effects influence the behavior of the tagged DIS cross section in the physical region but vanish at the pole [22]. Specifically, it is expected that in the region of the EMC effect $(x \gtrsim 0.3)$ the modifications of nucleon structure are proportional to the nucleon virtuality (or 4-momentum off-shellness) [51,52]. In tagged DIS, the virtuality of the active neutron is kinematically fixed by the momentum of the tagged proton and given by

$$
t^{\prime} \equiv\left(p_{p}-p_{d}\right)^{2}-m^{2}=-\frac{2\left(p_{p T}^{2}+a_{T}^{2}\right)}{\alpha_{p}} .
$$

The modification of the neutron's reduced cross section Eq. (29) can be approximately parametrized as

$$
\frac{\sigma_{\text {red }, n}\left(x_{n}, Q^{2}\right)[\text { bound }]}{\sigma_{\text {red }, n}\left(x_{n}, Q^{2}\right)[\text { free }]} \approx 1-\frac{2\left(p_{p T}^{2}+a_{T}^{2}\right)}{\alpha_{p}\left\langle t^{\prime}\right\rangle} f_{d}\left(x_{n}\right) .
$$

$\left\langle t^{\prime}\right\rangle$ is the "average nucleon virtuality" in the deuteron and has been estimated as $\approx-0.05 \mathrm{GeV}^{2}$ in nuclear structure 
calculations [52]. $f_{d}(x)$ is the function parametrizing the magnitude of the inclusive (untagged) EMC effect in the deuteron and has been estimated as $\left|f_{d}(x \gtrsim 0.3)\right| \lesssim 0.02$ using data on inclusive nuclear structure functions with $A>2$ and the $A$-dependence of the EMC effect [51,52]. When used in the impulse approximation formula (29), the modification (65) causes a few-percent change of the slope of the $p_{p T}^{2}$ dependence of the tagged cross section, with no change of the value at the pole position $p_{p T}^{2}=-a_{T}^{2}$. As explained above, this change does not affect the result of the extrapolation and does not introduce an additional uncertainty in the extraction of free nucleon structure. (For studies of the "tagged EMC" effect, where one interprets the data directly in the physical region $p_{p T}^{2}>0$ and aims to extract the nuclear modifications themselves, see Sec. VI and Ref. [39].)

Final-state interactions. Final-state interactions between the DIS products and the spectator nucleon influence the behavior of the tagged cross section in the physical region $p_{p T}^{2}\left(p_{n T}^{2}\right)>0$, but not the value at the pole. This is because the nucleon pole singularity is contained exactly in the "tree graph" of the impulse approximation, while "loop graphs" due to final-state interactions can only produce subleading singularities [22]. The BeAGLE physics model used in the present study is based on the impulse approximation and does not include final-state interactions. Theoretical calculations indicate that final-state interactions in tagged DIS at $x \gtrsim 0.1$ change the deuteron spectral function by $\lesssim 10 \%$ at $p_{p T}<100 \mathrm{MeV}$ and have a smooth dependence on $p_{p T}$. The inclusion of these effects would change the results in Fig. 8 by a relative amount $\lesssim 10 \%$, while being theoretically constrained to extrapolate to the same free neutron (proton) result. These effects therefore would not affect the quality of the fit or the uncertainty of the extrapolation. In this sense the use of the impulse approximation in the BeAGLE physics model is justified for the present purpose and does not represent a limitation; simulations of tagged DIS at low transverse momenta $p_{p T}\left(p_{n T}\right)<100 \mathrm{MeV}$ and nucleon structure extraction with pole extrapolation can be safely performed in this approximation. (We note that in studies of nuclear modifications in tagged DIS, where one measures at higher transverse momenta $p_{p T}\left(p_{n T}\right) \approx 300$ $600 \mathrm{MeV}$ [39] and interprets the data directly in the physical region, final-state interactions are generally large and need to be included explicitly in the physics model; see Sec. VI and Ref. [39].)

Target fragmentation. In tagged DIS we require the presence of a forward proton (neutron) with $\alpha_{p}\left(\alpha_{n}\right) \approx 1$ in the deuteron fragmentation region but have otherwise no information about the hadronic event (see Fig. 1). Such forward protons and neutrons can not only come from the spectator nucleon in the deuteron breakup but also from the target fragmentation of the active nucleon (baryon production at $x_{F} \approx$ -1 ). The two mechanisms cannot be distinguished event by event and should be treated jointly in the physics analysis. The target fragmentation nucleons have a broad $p_{T}^{2}$ distribution with a width $\left\langle p_{T}^{2}\right\rangle \approx 0.1-0.15 \mathrm{GeV}^{2}$ [23], much larger than the $p_{T}^{2}$ considered in the present study; see Sec. IV B. The target fragmentation mechanism does not have the nucleon pole of the spectator mechanism, so that the pole extrapolation procedure of Secs. II F and IV C eliminates the contribution of target fragmentation - another important advantage of this method.

Diffractive scattering. The present study of nucleon structure extraction from tagged DIS focuses on the region $x \gtrsim 0.1$. In DIS at $x \ll 0.1$, diffractive scattering becomes significant and constitutes $10-15 \%$ of the DIS cross section, as observed in measurements at HERA [53,54]. In diffractive events the nucleon remains intact and recoils with a typical momentum transfer $\approx$ few $100 \mathrm{MeV}$ (in the nucleon rest frame), and the other hadrons produced are separated by a rapidity gap. In tagged DIS on the deuteron, diffractive scattering on the nucleons (proton or neutron) creates several effects that are not included in the present simulations ${ }^{2}$ and require separate theoretical study: (i) The measurement cannot distinguish between the spectator nucleon and the diffractive nucleon (see above). (ii) The $p n$ state produced in diffractive DIS on the deuteron has small relative momentum $\approx$ few $100 \mathrm{MeV}$ and the same quantum numbers as the deuteron. There is a large amplitude for this state to remain a bound deuteron, resulting in coherent scattering. If a $p n$ breakup state is measured, its wave function will be strongly distorted by the requirement of orthogonality to the bound state (large final-state interactions). (iii) Interference between diffractive DIS on the proton and the neutron gives rise to nuclear shadowing [27,28]. These effects are the object of ongoing theoretical studies [55] and can be included of future simulations of tagged DIS at small $x$. Such measurements present a new opportunity to explore the dynamical origin of leading-twist nuclear shadowing [16], which is observed in hard exclusive processes with heavy nuclei [56-58] and governs the small- $x$ behavior of the nuclear PDFs and the approach to gluon saturation. In particular, such measurements could use double tagging-the detection of both the proton and the neutron resulting from the deuteron breakup-to completely fix the outgoing nucleonic configuration and enable a differential analysis of the interactions. Deuteron breakup in diffractive $J / \psi$ production at EIC was studied in Ref. [47].

\section{CONCLUSIONS}

We have performed a comprehensive study of deuteron DIS with spectator nucleon tagging at EIC, focusing on the extraction of free neutron and proton structure using the pole extrapolation method. Our framework combines theoretical methods of light-front nuclear structure, the BeAGLE $e A$ event generator, and a description of the EIC far-forward detector performance based on full GEANT4 simulations. We have simulated the measurement of the reduced cross sections and the extraction of free nucleon structure through pole extrapolation under realistic conditions, including detector acceptance and detector and beam effects on the far-forward momentum resolution. We have quantified the systematic uncertainties to the extent possible at the present stage and

\footnotetext{
${ }^{2}$ The present BeAGLE simulations include only the non-diffractive part of the DIS cross section. In the PYTHIA 6 parameters only Process 99 is selected.
} 
discussed possible refinements incorporating future developments. The main conclusions of the study are

(i) Detection of far-forward protons and neutrons in the momentum range needed for free nucleon structure extraction with spectator tagging, $\alpha_{p}\left(\alpha_{n}\right) \approx 1$ and $p_{p T}\left(p_{n T}\right) \lesssim 100 \mathrm{MeV}$, is possible with nearly full acceptance with the baseline EIC far-forward detector design.

(ii) The steep $p_{p T}\left(p_{n T}\right)$ dependence of the deuteron spectral function places high demands on the transverse momentum resolution in the tagged cross section measurement. The separation of deuteron and nucleon structure (pole removal) needs to be performed with binned $p_{p T}^{2}\left(p_{n T}^{2}\right)$ distributions corrected for detector performance. The overall detector resolution effects on the measured nucleon DIS cross section (before unfolding corrections) are estimated at $\approx 10 \%$ for proton tagging and $\approx 30 \%$ for neutron tagging. With unfolding corrections based on the actual EIC detector implementation, it is expected that a percent-level measurement of the tagged nucleon DIS cross section will be possible.

(iii) The pole extrapolation of the tagged nucleon DIS cross section can be performed with low-order polynomial fits and gives robust results. In the simulations these results can be validated by comparing with the physics model input.

(iv) Systematic uncertainties dominate the tagged deuteron cross section measurement and nucleon structure extraction in the DIS kinematics considered in the present study. The uncertainties arising from known sources (beam, detector, theory) are estimated at the few-percent level. More detailed estimates will become possible as the EIC detector design advances.

(v) The pole extrapolation method eliminates both initialstate nuclear modifications and final-state interactions in tagged DIS and minimizes the theoretical uncertainty in nucleon structure extraction. The only theoretical input is the asymptotic normalization constant of the $S$ state in the deuteron wave function, which is determined with high precision in low-energy nuclear structure calculations and measurements.

Altogether, tagged DIS measurements and free nucleon structure extraction appear feasible with the EIC accelerator and far-forward detector design, with an experimental accuracy that realizes the theoretical potential of the method.

\section{EXTENSIONS}

The present study has focused on the application of spectator tagging to inclusive DIS in typical EIC kinematics $(x \approx$ $10^{-2}-10^{-1}, Q^{2} \gtrsim 10 \mathrm{GeV}^{2}$ ) with the goal of extracting the free neutron and proton structure functions. The methods could be extended and applied to other high-energy processes with different physics goals:

Azimuthal angle dependence. The $\phi_{p}\left(\phi_{n}\right)$ dependence of the tagged DIS cross section provides interesting information on the light-front structure of the deuteron and the dynamics of the breakup process (the angle refers to the photon-deuteron collinear frame; see Sec. II A). T-even (time-reversal-even) azimuthal asymmetries such as $\cos \phi$ and $\cos 2 \phi$ are predicted by the impulse approximation, while T-odd asymmetries $[8,59]$ are proportional to final-state interactions and can provide sensitive tests of their dynamics (such structures can be formed with polarized electron and unpolarized deuteron beams) [41]. The $\phi$ dependence of the tagged DIS cross section also needs to be studied as a potential source of uncertainty in the measurement of the $\phi$-integrated cross sections, see Eqs. (18) and (49), in kinematic regions where the detector acceptance is effectively nonuniform in $\phi_{p}$ [60].

Flavor tagging with semi-inclusive DIS. Spectator nucleon tagging in deuteron DIS could be combined with measurements of semi-inclusive hadron production (pions, kaons) in the current fragmentation region of the active nucleon ("flavor tagging"). The possibility of measuring semi-inclusive DIS on the identified neutron as well as the proton would enable new studies of the nucleon flavor decomposition and the meson fragmentation functions, especially regarding unflavored and strange quark fragmentation [61]. Such measurements could be performed with the tagged neutron and proton in the deuteron as well as with the free proton; the comparison between the three would allow one to separate final-state interactions from initial-state structure. Even with the integrated luminosity $\approx 1 \mathrm{fb}^{-1}$ assumed in the present study, simultaneous binning in the spectator nucleon momentum and the semi-inclusive meson momentum should be possible. Such measurements would be attractive even without the full pole extrapolation selecting free nucleon configurations.

Exclusive processes. Spectator nucleon tagging in electron scattering on the deuteron could be used to measure hard exclusive processes on the neutron such as meson production and deeply virtual Compton scattering (DVCS) [4]. DVCS measurements on the neutron are important for probing the nucleon GPD $E$ appearing in the angular momentum sum rule, and for the flavor decomposition of the nucleon GPDs in general. In exclusive processes the active nucleon recoils with a momentum transfer $\approx$ few $100 \mathrm{MeV}$. When such processes occur in scattering on the deuteron, the recoiling active nucleon and the spectator experience strong final state interactions, which qualitatively change the spectator momentum distribution compared to the impulse approximation (similar to the case of diffractive DIS discussed in Sec. V B). Including these low-energy final-state interaction effects in the physics model and event generator is essential for realistic simulations of tagged exclusive processes. We note that the luminosity required for tagged exclusive processes is much more demanding than for tagged inclusive DIS, because the exclusive processes on the nucleon have low rates in themselves, and tagging with the deuteron further dilutes the statistics.

Nuclear modifications. Another class of applications of tagged DIS is the study of nuclear modifications of partonic structure (antishadowing at $x \approx 0.1$; EMC effect at $x \gtrsim 0.3$ ). These applications use tagging at higher spectator momenta $p_{p}\left(p_{n}\right) \approx 300-600 \mathrm{MeV}$ (in the deuteron rest frame) to select small-size configurations where the nucleons are strongly interacting and the dynamical modifications are expected to be 
large. At such spectator momenta final-state interactions are generally large and cause qualitative deviations from the impulse approximation. The analysis should focus on strategies to separate the effects of the initial-state modifications from those of final-state interactions, e.g., by using the different kinematic dependence of the effects. These measurements generally require higher luminosity than the extraction of free nucleon structure, because high-momentum tagging uses only a small fraction of the deuteron's momentum distribution, $\lesssim 1 \%$ for $p_{p}\left(p_{n}\right)>300 \mathrm{MeV}$. Simulations of tagged DIS at higher spectator momenta and the exploration of nuclear modifications with EIC will be reported in a forthcoming article [39].

Polarized deuteron. Polarized deuteron beams at EIC are regarded as technically possible and considered as a future option [18]. This would open the possibility of performing measurements of DIS and other high-energy processes on the polarized deuteron with spectator nucleon tagging. The measured spectator momentum controls the $D / S$ wave ratio in the deuteron and thus fixes the spin structure of the $p n$ configuration during the high-energy process. This feature can be used to eliminate $D$-wave depolarization in the extraction of neutron spin structure, or to maximize the $D$ wave in the exploration of vector- or tensor-polarized spin asymmetries [24-26]. The luminosity and polarization requirements of such measurements are under investigation [19,62]. A study of polarized DIS on ${ }^{3} \mathrm{He}$ with spectator tagging at EIC has been reported in Ref. [63]. We note that, in the present EIC design, electron polarization is available only in configurations with center-of-mass energies above $29 \mathrm{GeV} /$ nucleon, because of limitations on the minimal electron beam energy [18].

\section{ACKNOWLEDGMENTS}

The authors would like to thank Mark Baker and Elke Aschenauer for valuable discussions on the BeAGLE event generator and DIS physics; Mark Strikman, Vadim Guzey, and Wim Cosyn, for collaboration and discussions on the theoretical treatment of deuteron structure and tagged DIS; and Rocco Schiavilla, for helpful communication on low-energy deuteron structure. The authors would also like to thank the EIC Project's Interaction Region Working Group for their help in understanding the various impacts of the IR design. The work of A.J. is supported by the US Department of Energy under Award No. DE-SC0012704. The work of Z.T. is supported by Brookhaven National Laboratory's Laboratory Directed Research and Development Program under Award LDRD-039; by the US Department of Energy under Award No. DE-SC0012704, and by the Goldhaber Distinguished Fellowship at Brookhaven National Laboratory. The work of C.W. is supported by the US Department of Energy, Office of Science, Office of Nuclear Physics, under Contract No. DE-AC05-06OR23177.

\section{APPENDIX A: DEUTERON STRUCTURE MODEL}

\section{Light-front spectral function}

In this Appendix we describe the elements of the deuteron structure model used in the event generation and physics analysis in the present study. This includes the construction of the deuteron light-front wave function and spectral function, the nonrelativistic approximation, the nucleon pole and its parameters, and a minimal two-pole model of the wave function. These materials can be used in simulations of other high-energy scattering processes with deuteron breakup. Further information can be found in Refs. [23,24].

In the light-front description of deuteron structure the $p n$ configurations are characterized by the proton light-front momentum variables $\alpha_{p}$ and $\boldsymbol{p}_{p T}$; see Eq. (19). An alternative set of variables is the proton 3-momentum $\boldsymbol{k}$ in the center-ofmass $(\mathrm{CM})$ momentum of the $p n$ configuration. The relation between the variables is

$$
\alpha_{p}=1+\frac{k^{z}}{E(\boldsymbol{k})}, \quad \boldsymbol{p}_{p T}=\boldsymbol{k}_{T}
$$

or, inversely,

$$
k^{z}=E(\boldsymbol{k})\left(\alpha_{p}-1\right), \quad \boldsymbol{k}_{T}=\boldsymbol{p}_{p T},
$$

where

$$
E(\boldsymbol{k}) \equiv \sqrt{|\boldsymbol{k}|^{2}+m_{N}^{2}}=\left[\frac{\left|\boldsymbol{p}_{p T}\right|^{2}+m_{N}^{2}}{\alpha_{p}\left(2-\alpha_{p}\right)}\right]^{1 / 2}
$$

is the nucleon energy in the CM frame. The integration measures in the variables are related as

$$
\frac{d \alpha_{p} d^{2} p_{p T}}{\alpha_{p}\left(2-\alpha_{p}\right)}=\frac{d^{3} k}{E(\boldsymbol{k})} .
$$

The use of the CM momentum variable provides a rotationally symmetric representation of light-front quantum mechanics in the two-body sector. The deuteron light-front wave function is represented in terms of a rotationally symmetric wave function as

$$
\Psi_{d}\left(\alpha_{p}, \boldsymbol{p}_{p T}\right)=\widetilde{\Psi}_{d}(\boldsymbol{k}),
$$

with the normalization condition [see Eqs. (24) and (A4)]

$$
\begin{gathered}
\int \frac{d \alpha_{p} d^{2} p_{p T}}{\alpha_{p}\left(2-\alpha_{p}\right)}\left|\Psi_{d}\left(\alpha_{p}, \boldsymbol{p}_{p T}\right)\right|^{2} \\
=\int \frac{d^{3} k}{E(\boldsymbol{k})}\left|\widetilde{\Psi}_{d}(\boldsymbol{k})\right|^{2}=1
\end{gathered}
$$

The light-front spectral function, which appears in the description of high-energy scattering processes on the deuteron in the impulse approximation, is defined in terms of the lightfront wave function by Eq. (27),

$$
\mathcal{S}_{d}\left(\alpha_{p}, \boldsymbol{p}_{p T}\right) \equiv \frac{\left|\Psi_{d}\left(\alpha_{p}, \boldsymbol{p}_{p T}\right)\right|^{2}}{2-\alpha_{p}} .
$$

This function is related in a simple way to the density of the rotationally symmetric wave function. Using Eqs. (A5) and (A4) one obtains

$$
\begin{aligned}
\mathcal{S}_{d} & \left(\alpha_{p}, \boldsymbol{p}_{p T}\right)\left[2(2 \pi)^{3}\right] d \Gamma_{p} \\
= & \left|\Psi_{d}\left(\alpha_{p}, \boldsymbol{p}_{p T}\right)\right|^{2} \frac{d \alpha_{p} d^{2} p_{p T}}{\alpha_{p}\left(2-\alpha_{p}\right)} \\
= & \left|\widetilde{\Psi}_{d}(\boldsymbol{k})\right|^{2} \frac{d^{3} k}{E(\boldsymbol{k})} .
\end{aligned}
$$


Thus the product of the light-front spectral function and the light-front phase space element is equal to the product of the rotationally symmetric momentum distribution and its phase space element. This illustrates how rotational invariance is recovered in the light-front description and allows one to make connection with the nonrelativistic theory. Using the correspondence Eq. (A8), the reduced cross section of tagged DIS in the impulse approximation, Eq. (29), can be expressed in terms of the CM momentum variable as

$$
\begin{aligned}
& \bar{\sigma}_{\text {red }, d}\left(x, Q^{2} ; \alpha_{p}, p_{p T}\right) d \Gamma_{p} \\
& =\sigma_{\text {red }, n}\left(x_{n}, Q^{2}\right)\left[2(2 \pi)^{3}\right] \mathcal{S}_{d}\left(\alpha_{p}, p_{p T}\right) d \Gamma_{p} \\
& =\sigma_{\text {red }, n}\left(x_{n}, Q^{2}\right)\left|\widetilde{\Psi}_{d}(\boldsymbol{k})\right|^{2} \frac{d^{3} k}{E(\boldsymbol{k})} .
\end{aligned}
$$

\section{Proton and neutron momenta}

In the light-front description the proton and neutron lightfront momenta in each $p n$ configuration add up to the total light-front momentum of the deuteron bound state. The neutron light-front momentum in a configuration with proton light-front momentum $\alpha_{p}$ and $\boldsymbol{p}_{p T}$ is given by

$$
\alpha_{n}=2-\alpha_{p}, \quad \boldsymbol{p}_{n T}=-\boldsymbol{p}_{p T} .
$$

The deuteron light-front wave function is symmetric under the exchange of proton and neutron variables (isospin symmetry):

$$
\Psi_{d}\left(\alpha_{n}, \boldsymbol{p}_{n T}\right)=\Psi_{d}\left(2-\alpha_{p},-\boldsymbol{p}_{p T}\right)=\Psi_{d}\left(\alpha_{p}, \boldsymbol{p}_{p T}\right) .
$$

The wave function can therefore equivalently be regarded as a function of the neutron momentum; because

$$
\int \frac{d \alpha_{p} d^{2} p_{p T}}{\alpha_{p}\left(2-\alpha_{p}\right)}[\cdots]=\int \frac{d \alpha_{n} d^{2} p_{n T}}{\alpha_{n}\left(2-\alpha_{n}\right)}[\cdots],
$$

the normalization condition Eq. (A6) takes the same form in the neutron variables. In terms of the $\mathrm{CM}$ momentum variable the neutron has momentum $-\boldsymbol{k}$, and the wave function satisfies

$$
\widetilde{\Psi}_{d}(-\boldsymbol{k})=\widetilde{\Psi}_{d}(\boldsymbol{k})
$$

In high-energy scattering on the deuteron with neutron tagging, the impulse approximation involves the spectral function

$$
\mathcal{S}_{d}\left(\alpha_{n}, \boldsymbol{p}_{n T}\right) \equiv \frac{\left|\Psi_{d}\left(\alpha_{n}, \boldsymbol{p}_{n T}\right)\right|^{2}}{2-\alpha_{n}},
$$

where $\alpha_{n}$ and $\boldsymbol{p}_{n T}$ are the light-front momentum variables of the detected neutron; Eq. (A14) is the same mathematical function as the spectral function for proton tagging, Eq. (A7), only evaluated at the neutron variables $\alpha_{n}$ and $\boldsymbol{p}_{n T}$. Note that the spectral function is not symmetric under the exchange of proton and neutron momenta,

$$
\mathcal{S}_{d}\left(2-\alpha_{p},-\boldsymbol{p}_{p T}\right) \neq \mathcal{S}_{d}\left(\alpha_{p}, \boldsymbol{p}_{p T}\right),
$$

because the definition (A7) contains the flux factor $1 /(2-$ $\left.\alpha_{p}\right)$. However, the product of the spectral function and the phase space element is symmetric under proton-neutron exchange,

$$
\begin{aligned}
\mathcal{S}_{d} & \left(\alpha_{p}, \boldsymbol{p}_{p T}\right)\left[2(2 \pi)^{3}\right] d \Gamma_{p} \\
= & \left|\Psi_{d}\left(\alpha_{p}, \boldsymbol{p}_{p T}\right)\right|^{2} \frac{d \alpha_{p} d^{2} p_{p T}}{\alpha_{p}\left(2-\alpha_{p}\right)} \\
= & \left|\Psi_{d}\left(\alpha_{n}, \boldsymbol{p}_{n T}\right)\right|^{2} \frac{d \alpha_{n} d^{2} p_{n T}}{\alpha_{n}\left(2-\alpha_{n}\right)} \\
= & \mathcal{S}_{d}\left(\alpha_{n}, \boldsymbol{p}_{n T}\right)\left[2(2 \pi)^{3}\right] d \Gamma_{n} .
\end{aligned}
$$

This can also be seen directly from the expression in terms of the $\boldsymbol{k}$-vector variable, Eq. (A8), which is symmetric under $\boldsymbol{k} \rightarrow-\boldsymbol{k}$.

The treatment of deuteron structure described here assumes isospin symmetry and neglects the difference between the proton and neutron masses. The nucleon mass in Eq. (A3) is defined as the average of the physical proton and neutron masses,

$$
m_{N} \equiv \frac{1}{2}\left(m_{p}+m_{n}\right)
$$

With this definition the relation between the deuteron mass and the binding energy takes the form

$$
M_{d}=m_{p}+m_{n}-\epsilon_{d}=2 m_{N}-\epsilon_{d},
$$

i.e., the use of the average nucleon mass preserves the relation between the physical deuteron mass and the binding energy. This circumstance is important when matching the deuteron structure model with simulation codes that use the exact proton and neutron masses.

\section{Spin degrees of freedom}

For reference we want to summarize also the treatment of the deuteron and nucleon spin degrees of freedom. The deuteron light-front wave function depends on the light-front helicity variables of the deuteron and the nucleons, $\lambda_{d}=$ $\{1,0,-1\}$ and $\lambda_{p, n}=\left\{\frac{1}{2},-\frac{1}{2}\right\}$ :

$$
\Psi_{d}\left(\alpha_{p}, \boldsymbol{p}_{p T} ; \lambda_{p}, \lambda_{n} \mid \lambda_{d}\right)
$$

The representation in terms of the CM momentum variable, Eq. (A5), exhibits the underlying rotational invariance of the light-front wave function and allows one to infer its spin structure from that of the rotationally invariant wave function. It is given by

$$
\begin{aligned}
\Psi_{d}\left(\alpha_{p}, \boldsymbol{p}_{p T} ; \lambda_{p}, \lambda_{n} \mid \lambda_{d}\right)= & \sum_{\lambda_{p}^{\prime}, \lambda_{n}^{\prime}} \widetilde{\Psi}_{d}\left(\boldsymbol{k}, \lambda_{n}^{\prime}, \lambda_{p}^{\prime} \mid \lambda_{d}\right) \\
& \times U^{*}\left(\boldsymbol{k}, \lambda_{p}^{\prime}, \lambda_{p}\right) U^{*}\left(-\boldsymbol{k}, \lambda_{n}^{\prime}, \lambda_{n}\right),
\end{aligned}
$$

where $\lambda_{p, n}^{\prime}$ are the canonical spin variables and $U^{*}$ are the Melosh rotations connecting the light-front helicity with the canonical spin variables, whose explicit form is given in Ref. [24] and is not needed here (the deuteron light-front helicity is the same as its canonical spin because $\boldsymbol{p}_{T d}=0$ ). 
The normalization condition including spins is

$$
\begin{aligned}
& \sum_{\lambda_{p}, \lambda_{n}} \int \frac{d \alpha_{p} d^{2} p_{p T}}{\alpha_{p}\left(2-\alpha_{p}\right)} \Psi_{d}^{*}\left(\alpha_{p}, \boldsymbol{p}_{p T} ; \lambda_{p}, \lambda_{n} \mid \lambda_{d}^{\prime}\right) \\
& \quad \times \Psi_{d}\left(\alpha_{p}, \boldsymbol{p}_{p T} ; \lambda_{p}, \lambda_{n} \mid \lambda_{d}\right) \\
& =\sum_{\lambda_{p}^{\prime}, \lambda_{n}^{\prime}} \int \frac{d^{3} k}{E(\boldsymbol{k})} \widetilde{\Psi}_{d}^{*}\left(\boldsymbol{k}, \lambda_{n}^{\prime}, \lambda_{p}^{\prime} \mid \lambda_{d}\right) \widetilde{\Psi}_{d}\left(\boldsymbol{k}, \lambda_{n}^{\prime}, \lambda_{p}^{\prime} \mid \lambda_{d}\right) \\
& =\delta\left(\lambda_{d}^{\prime}, \lambda_{d}\right) .
\end{aligned}
$$

The unpolarized deuteron spectral function, Eqs. (27) and (A7), is defined as the sum over the nucleon light-front helicities and the average over the deuteron light-front helicity,

$$
S_{d}\left(\alpha_{p}, \boldsymbol{p}_{p T}\right) \equiv \frac{1}{3} \sum_{\lambda_{d}} \sum_{\lambda_{p}, \lambda_{n}} \frac{\left|\Psi_{d}\left(\alpha_{p}, \boldsymbol{p}_{p T} ; \lambda_{p}, \lambda_{n} \mid \lambda_{d}\right)\right|^{2}}{2-\alpha_{p}}
$$

The expression in Eq. (A8) becomes

$$
[\cdots]=\frac{1}{3} \sum_{\lambda_{d}} \sum_{\lambda_{p}^{\prime}, \lambda_{n}^{\prime}}\left|\widetilde{\Psi}_{d}\left(\boldsymbol{k}, \lambda_{n}^{\prime}, \lambda_{p}^{\prime} \mid \lambda_{d}\right)\right|^{2} \frac{d^{3} k}{E(\boldsymbol{k})} .
$$

The rotationally symmetric wave function can be decomposed into $S$ - and $D$-wave components with orbital angular momenta $L=0$ and 2 in the $\boldsymbol{k}$ vector variable and corresponding spin structure [24]

$$
\widetilde{\Psi}_{d}\left(\boldsymbol{k}, \lambda_{n}^{\prime}, \lambda_{p}^{\prime} \mid \lambda_{d}\right) \rightarrow \widetilde{\Psi}_{d}[L=0]+\widetilde{\Psi}_{d}[L=2] .
$$

The $S$ and $D$ waves of the light-front wave function then follow from Eq. (A20). The $D$ wave contributes to the unpolarized spectral function only at large nucleon momenta $\left|\boldsymbol{p}_{p, n}\right| \gtrsim 200 \mathrm{MeV}$ (in the deuteron rest frame), which are not used in the present study of free nucleon structure extraction; the only role of the $D$ wave in this context is its contribution to the normalization of the wave function, which balances that of the $S$ wave (see below).

In the text of this article and in the rest of this Appendix we suppress the spin degrees of freedom for brevity. The expressions of the unpolarized spectral function and the impulse approximation cross section are always understood in the sense of Eq. (A22).

\section{Nonrelativistic approximation}

The representation in terms of the $\mathrm{CM}$ momentum variable can be used to construct an approximation to the lightfront wave function in terms of the nonrelativistic deuteron wave function. The nonrelativistic wave function $\Phi_{d}(\boldsymbol{p})$ is a function of the ordinary proton 3-momentum $\boldsymbol{p} \equiv \boldsymbol{p}_{p}$ in the deuteron rest frame with normalization

$$
\int d^{3} p\left|\Phi_{d}(\boldsymbol{p})\right|^{2}=1
$$

An approximate relativistic wave function is provided by

$$
\widetilde{\Psi}_{d}(\boldsymbol{k}) \stackrel{\text { app }}{=} \sqrt{E(\boldsymbol{k})} \Phi_{d}(\boldsymbol{k}) .
$$

The nonrelativistic wave function $\Phi_{d}$ on the right-hand side is evaluated at the momentum variable $\boldsymbol{k}$ that is the argument of the relativistic wave function $\widetilde{\Psi}_{d}$ on the left-hand side.
The factor $\sqrt{E(\boldsymbol{k})}$ ensures that $\widetilde{\Psi}_{d}$ obeys the normalization condition (A6) if $\Phi_{d}$ obeys Eq. (A25).

The nonrelativistic approximation is well justified and adequate at nucleon momenta $\left|\boldsymbol{p}_{p, n}\right| \lesssim 200 \mathrm{MeV}$ (in the deuteron rest frame), as are considered in the present study of lowmomentum spectator tagging. In particular, the approximation (A26) implements the analytic properties of the wave function at small momenta and the nucleon pole, which play an essential role in free nucleon structure extraction (see Sec. IIF). More generally, the approximation in Eq. (A26) allows one to recruit the extensive results on deuteron structure from non-relativistic nuclear theory (with realistic $N N$ interactions) for the description of light-front structure and high-energy scattering processes.

\section{Nucleon pole}

The rotationally invariant representation establishes the analytic properties of the deuteron light-front wave function and exhibits the nucleon pole, which dominates the behavior of the wave function at low momenta and plays an essential role in nucleon structure extraction. The pole occurs in the $S$ wave of the nonrelativistic wave function and is of the form

$$
\Phi_{d}(\boldsymbol{p})[L=0] \approx \frac{\Gamma}{|\boldsymbol{p}|^{2}+a^{2}},
$$

where the pole position is given by

$$
a^{2} \equiv \epsilon_{d} m_{N}
$$

and $\Gamma$ denotes the residue. In the relativistic wave function obtained from Eq. (A26) the pole is of the form

$$
\widetilde{\Psi}_{d}(\boldsymbol{k})[L=0] \approx \frac{\sqrt{m_{N}} \Gamma}{|\boldsymbol{k}|^{2}+a^{2}},
$$

where the factor $\sqrt{E(\boldsymbol{k})}$ at the pole has been approximated by its value at $|\boldsymbol{k}|^{2}=0, \sqrt{m_{N}}$ (corrections are $\approx \epsilon_{d} / m_{N}$ and negligible). Substituting $\left|\boldsymbol{k}^{2}\right|$ in Eq. (A29) by its expression in terms of the light-front momentum variables, Eqs. (A2) and (A3), one obtains the analytic structure of the light-front wave function as in Eqs. (40), (41), and (43):

$$
\begin{aligned}
\Psi_{d}\left(\alpha_{p}, \boldsymbol{p}_{p T}\right) & \approx \frac{R\left(\alpha_{p}\right)}{\left|\boldsymbol{p}_{p T}\right|^{2}+a_{T}^{2}\left(\alpha_{p}\right)}, \\
a_{T}^{2}\left(\alpha_{p}\right) & =\left(\alpha_{p}-1\right)^{2} m_{N}^{2}+\alpha_{p}\left(2-\alpha_{p}\right) a^{2}, \\
R\left(\alpha_{p}\right) & =\alpha_{p}\left(2-\alpha_{p}\right) \sqrt{m_{N}} \Gamma .
\end{aligned}
$$

This analytic structure is used for the pole extrapolation in $\left|\boldsymbol{p}_{p T}\right|^{2}$ at fixed $\alpha_{p}$ in Sec. II F.

The nucleon pole in the nonrelativistic momentum-space wave function (A27) determines the large-distance behavior of the corresponding coordinate-space wave function. The residue $\Gamma$ is related to the so-called asymptotic normalization constant of the $S$-state wave function, $A_{S}$. The nonrelativistic coordinate-space wave function is defined as

$$
\begin{aligned}
\Phi_{d}(\boldsymbol{r}) & \equiv \int \frac{d^{3} p}{(2 \pi)^{3}} e^{i p r} \Phi_{d}(\boldsymbol{p}), \\
\int d^{3} r\left|\Phi_{d}(\boldsymbol{r})\right|^{2} & =1 .
\end{aligned}
$$


The asymptotic behavior of the $S$-state radial wave function is of the form

$$
\Phi_{d}(\boldsymbol{r})[L=0] \approx \frac{A_{S}}{\sqrt{4 \pi}} \frac{e^{-a r}}{r} \quad(r \rightarrow \infty) .
$$

The exponential decay at large distances is determined by the scale derived from the pole position of the momentum-space wave function, Eqs. (A27) and (A28):

$$
a^{-1}=(45 \mathrm{MeV})^{-1}=4.3 \mathrm{fm},
$$

known as the Bethe-Peierls radius of the deuteron. The relation between the constant $A_{S}$ and the residue $\Gamma$ is

$$
A_{S}=\sqrt{2} \pi \Gamma \text {. }
$$

The asymptotic normalization constant $A_{S}$ is measured in low-energy deuteron breakup reactions [64]. It can also be determined with high precision from deuteron bound state calculations using empirical $N N$ potentials [65] or effective field theory (EFT) controlled interactions [66]. This information can be used to determine the value and uncertainty of the residue $\Gamma$ needed for the pole extrapolation high-energy scattering. For reference, a compilation of the values of $A_{S}$ and $\Gamma$ from various sources is provided in Table II.

The BeAGLE MC generator uses the parametrization of the deuteron momentum density of Ref. [48] for evaluating the tagged DIS cross section via Eqs. (A7) and (A8) (present code version 1.0; see Sec. III A). For reference we quote here the nucleon pole parameters corresponding to this parametrization. The parametrization is provided by Eq. (74) of Ref. [48], for $A=2$, with the input parameters given in Table A.1 of Ref. [48]: $A_{1}^{(0)}=157.4 \mathrm{fm}^{3}, B_{1}^{(0)}=$ $1.24 \mathrm{fm}^{2}, C_{1}^{(0)}=18.3 \mathrm{fm}^{2}$. In terms of these parameters the pole parameters in our convention Eq. (A27) are obtained as

$$
\begin{aligned}
a^{2} & =\left[C_{1}^{(0)}\right]^{-1}=0.002121 \mathrm{GeV}^{2}, \\
\Gamma^{2} & =\frac{A_{1}^{(0)} \exp \left[B_{1}^{(0)} / C_{1}^{(0)}\right]}{4 \pi\left[C_{1}^{(0)}\right]^{2}}=0.007885 \mathrm{GeV} .
\end{aligned}
$$

While the precise numerical values have no physical significance, they can be used for the numerical validation of the pole removal procedure in BeAGLE simulations as performed in the present study. Because the values of $a^{2}$ and $\Gamma$ used in various deuteron structure models show variations at the percent level (see Table II), one should use exactly the values (A38) and (A39) in pole removal studies with BeAGLE, to avoid artifacts from mismatched parameters.

\section{Two-pole parametrization}

A minimal parametrization of the deuteron wave function is obtained by supplementing the nucleon pole in the $S$ state with a second "effective" pole, which effectively accounts for the high-momentum components (two-pole parametrization, or Hulthen wave function). It is of the form

$$
\Phi_{d}(\boldsymbol{p})_{\mathrm{two}-\mathrm{pole}}=\frac{1}{\sqrt{c}}\left(\frac{1}{|\boldsymbol{p}|^{2}+a^{2}}-\frac{1}{|\boldsymbol{p}|^{2}+b^{2}}\right),
$$

where $a$ is the nucleon pole position (A28), $b$ is the position of the effective pole, and $c$ is fixed by the normalization
TABLE II. Values of the asymptotic $S$-state normalization constant in the deuteron nonrelativistic wave function, $A_{S}$, Eq. (A35), and the corresponding values of the residue of the nucleon pole, $\Gamma$, Eqs. (A27) and (A37), obtained from experiments or theoretical models of deuteron structure. References [65] and [66] are theoretical calculations based on $N N$ interactions; Ref. [48] and the two-pole parametrization are empirical parametrizations of the deuteron momentum density.

\begin{tabular}{lll}
\hline \hline Source & $A_{S}\left(\mathrm{fm}^{-1 / 2}\right)$ & $\Gamma\left(\mathrm{GeV}^{1 / 2}\right)$ \\
\hline Experiment [64] & $0.8781(44)$ & $0.08772(44)$ \\
AV18 [65] & 0.8850 & 0.0885 \\
EFTa [66] & 0.8777 & 0.0877 \\
EFTb [66] & 0.8904 & 0.0890 \\
EFTc [66] & 0.8964 & 0.0896 \\
CS1995 [48] & 0.888 & 0.0888 \\
Two-pole, Eq. (A42) & 0.885 & 0.0885 \\
\hline \hline
\end{tabular}

condition (A25) as

$$
c=\frac{\pi^{2}(a-b)^{2}}{a b(a+b)} .
$$

The residue of the nucleon pole in Eq. (A40) is given by

$$
\Gamma=1 / \sqrt{c} \text {. }
$$

The value of $a$ is calculated from Eq. (A28) using $m_{N}=0.939$ $\mathrm{GeV}$ and $\epsilon_{d}=2.23 \mathrm{MeV}$ :

$$
a=\sqrt{m_{N} \epsilon_{d}}=0.04576 \mathrm{GeV} .
$$

The value of $b$ is fixed empirically as [67]

$$
b=0.2719 \mathrm{GeV} \text {. }
$$

The parametrization (A40) embodies the correct analytic properties of the deuteron wave function at small momenta. The position and residue of the nucleon pole are given explicitly in terms of the model parameters; see Eq. (A42). With the parameter values of Eqs. (A43) and (A44), the value of $\Gamma$ in the two-pole parametrization agrees with the AV18 result within $\ll 1 \%$ (see Table II). The two-pole parametrization also provides an excellent approximation to the unpolarized deuteron momentum density obtained with realistic wave functions up to $|\boldsymbol{p}| \approx 300 \mathrm{MeV}$. Note that the two-pole parametrization uses only the $S$ wave, while realistic wave functions have $S$ and $D$ waves; the unpolarized momentum densities are nevertheless very close because the $S$ wave in the two-pole model is larger than that in realistic models at $|\boldsymbol{p}| \approx$ few $100 \mathrm{MeV}$ (it does not have a node) and makes up for the missing $D$-wave strength. As such the two-pole parametrization is fully adequate for unpolarized deuteron tagging at low momenta $|\boldsymbol{p}| \lesssim 100 \mathrm{MeV}$ and can be used for analytic and numerical studies of pole extrapolation.

\section{APPENDIX B: DETECTOR SIMULATIONS}

\section{Geometric acceptance}

In this Appendix we summarize the results of the full detector simulations that were used to quantify the EIC farforward acceptance and the detector and beam effects on the 

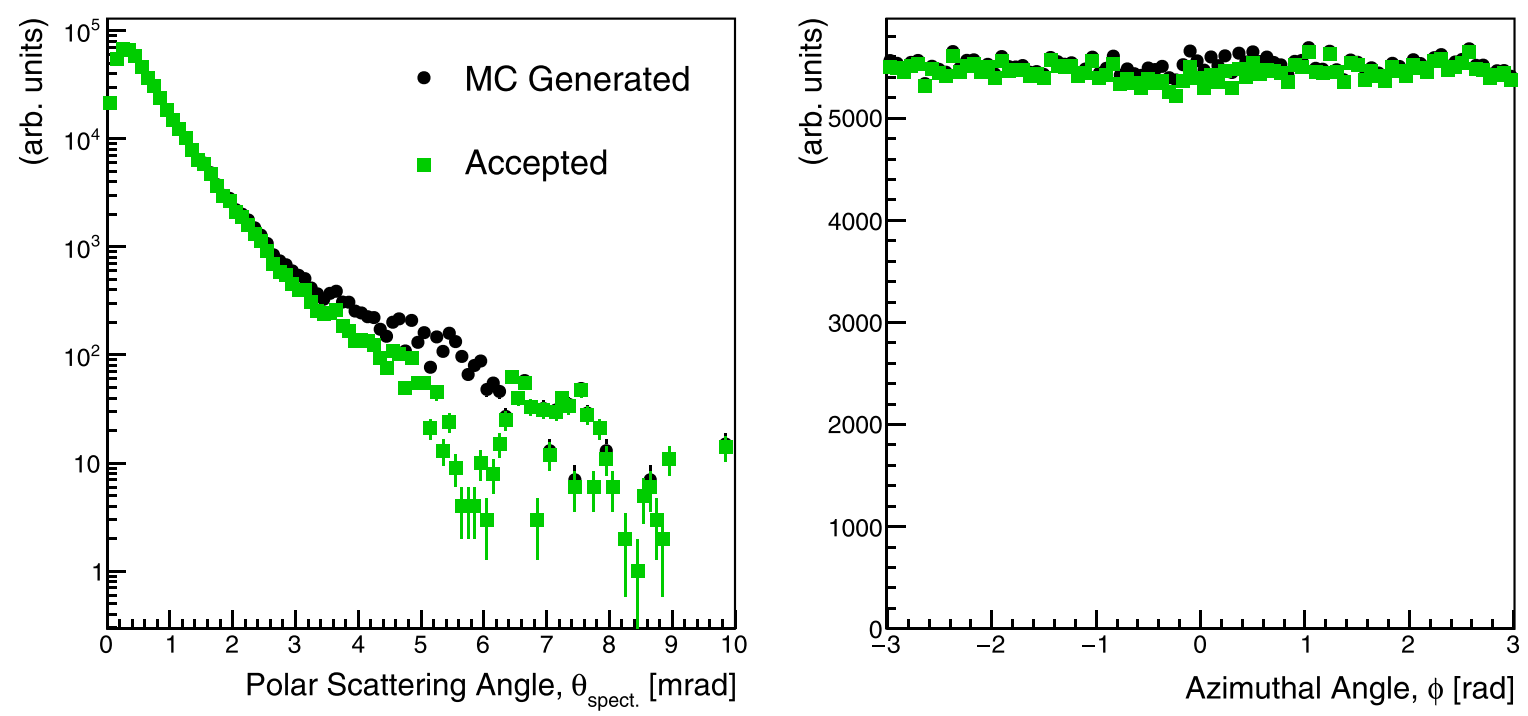

FIG. 12. Geometric acceptances in polar angle, $\theta_{p}$ (left), and azimuthal angle, $\phi_{p}$ (right) for the spectator protons. In the graphs the proton angles are denoted by $\theta_{\text {spect }} \equiv \theta_{p}$ and $\phi \equiv \phi_{p}$.

momentum resolution. The simulations were performed by processing a subsample of the BeAGLE tagged DIS events with the EICROOT framework [49], which implements the far-forward detectors in GEANT4 [50] in the configuration specified in the EIC Yellow Report [19]. In the physics study in the main text, the acceptance and resolution were described by parametrizations based on these simulation results. The simulation results summarized here document these parametrizations, provide additional insight into the detector performance (e.g., the relative contribution of various effects on the momentum resolution), and can be used in similar physics studies.

The geometric acceptances were simulated with the full implementation of the EIC interaction region and the farforward detectors (see Fig. 4), including the geometry/size of the elements, the dipole and quadrupole fields of the optics, and the transport of charged particles with magnetic rigidities different from that of the beam. Figure 12 shows the geometric acceptance for spectator protons in a deuteron beam with $110 \mathrm{GeV} /$ nucleon energy, as used in the present study. One observes that protons are fully accepted by the detector up to $\approx 2.8 \mathrm{mrad}$ in polar angle, at which point protons at $\phi=0 \mathrm{rad}$ begin to be lost in the quadrupole magnets due to their lower rigidity compared to the beam. The gap seen between 5 and $6 \mathrm{mrad}$ is the transition region between the Roman pots or off-momentum detector acceptance and the acceptance of the B0 spectrometer detector.

Figure 13 shows the corresponding acceptance for spectator neutrons. One sees that the neutron cone is detected with azimuthally symmetric acceptance up to $4.0 \mathrm{mrad}$, and
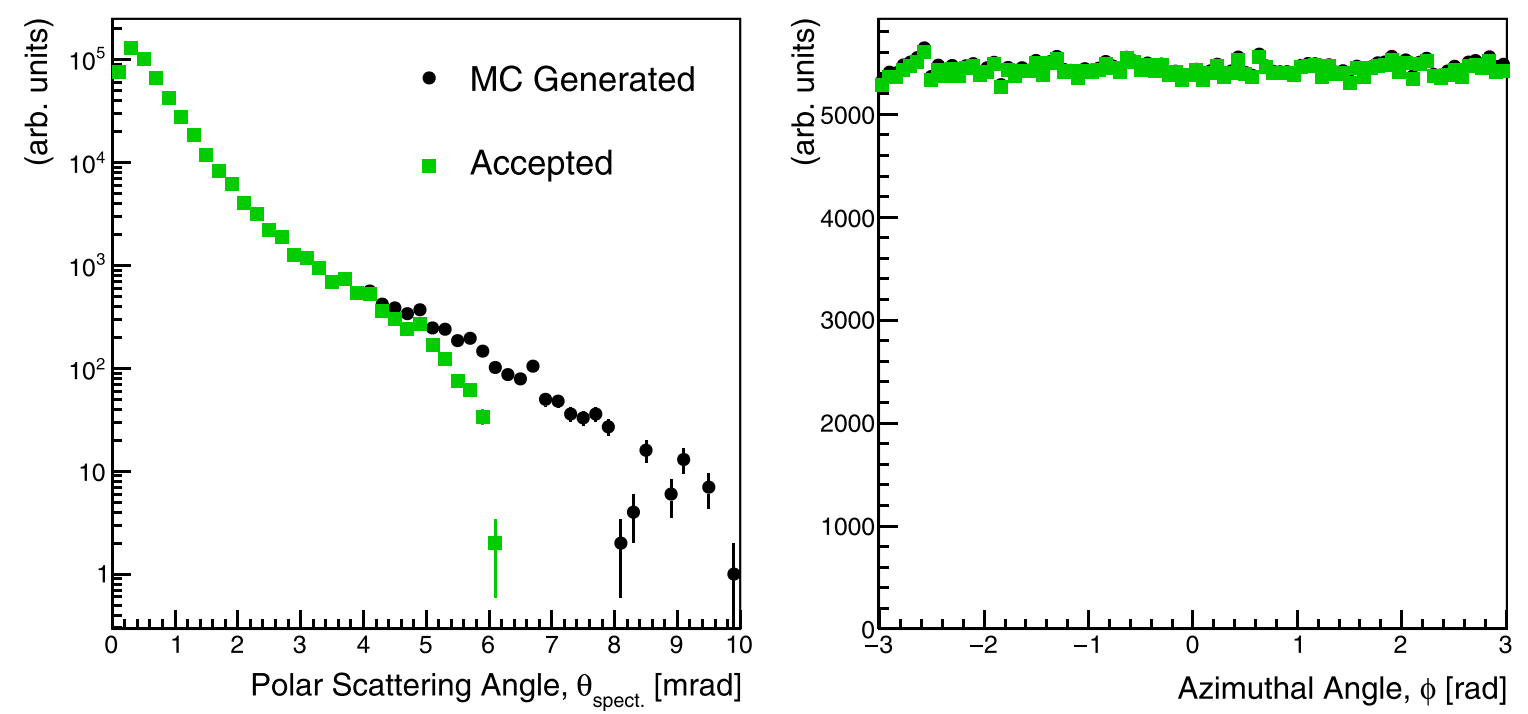

FIG. 13. Geometric acceptances in polar angle, $\theta_{n}$ (left), and azimuthal angle, $\phi_{n}$ (right) for the spectator neutrons. In the graphs the neutron angles are denoted by $\theta_{\text {spect }} \equiv \theta_{n}$ and $\phi \equiv \phi_{n}$. 

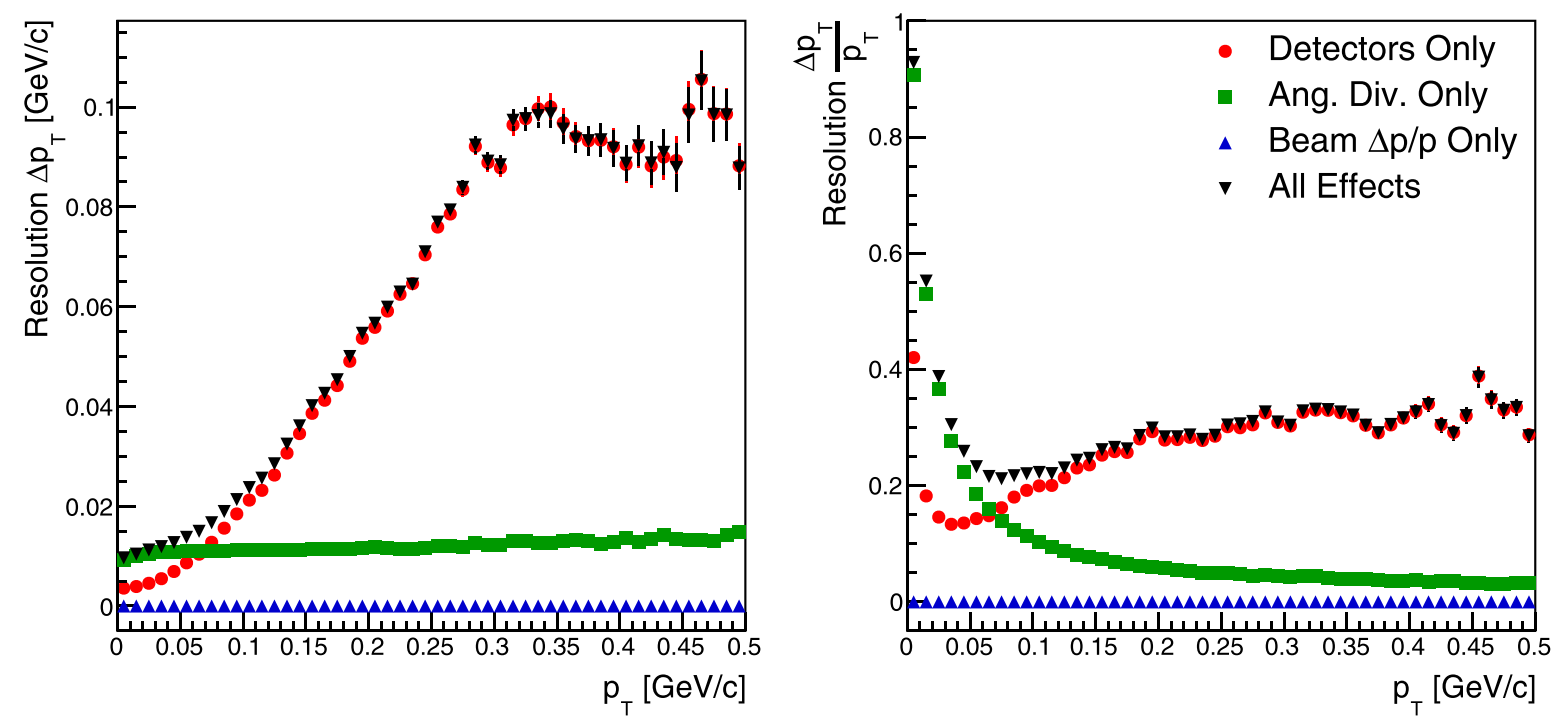

FIG. 14. Absolute (left) and relative (right) transverse momentum resolution for spectator protons obtained from the simulations. The plots show the contributions of the various effects and the total resolution.

acceptance up to $\approx 5.5 \mathrm{mrad}$ for $\phi=0 \mathrm{rad}$. For the neutron spectators in the deuteron at $110 \mathrm{GeV} /$ nucleon energy, the overwhelming majority are able to reach the detector, with the acceptance being $100 \%$ for the kinematics relevant for pole extrapolation. The addition of the beam pipe, which is still being designed at the time of this analysis, will reduce the overall efficiency by 5-20\%, depending on the degree of optimization employed in the design (exit window, material, etc.). A study of a simplistic beam pipe design and its associated impact are discussed in Ref. [68].

\section{Momentum resolution}

The resolutions were measured using full GEANT4 simulations including effects of detector-level reconstruction (e.g., finite pixel size, energy resolution) and effects related to the beam, which are modeled by smearing the final-state par- ticle vectors. The angular divergence smearing is included by taking the RMS angular divergence $\Delta \theta_{x, y}$ values from Table 3.4 in the EIC Conceptual Design Report [18] as the widths $(\sigma)$ for random Gaussian smearing in both $p_{x}$ and $p_{y}$, with a mean of zero (assuming the beams would have no transverse components of momentum without the divergence). Then, the final-state particle vector is boosted to the rest frame of the unsmeared deuteron (ion rest frame), which has only a longitudinal momentum component. The particle vector is then boosted back to the laboratory frame, but using a boost vector from the deuteron beam vector now containing the randomly smeared $p_{x}$ and $p_{y}$ components, simulating the effect of having a deuteron beam with initial transverse momentum components being carried to the final-state particle vectors in the laboratory frame. This approach only applies reconstruction smearing to the transverse momentum.
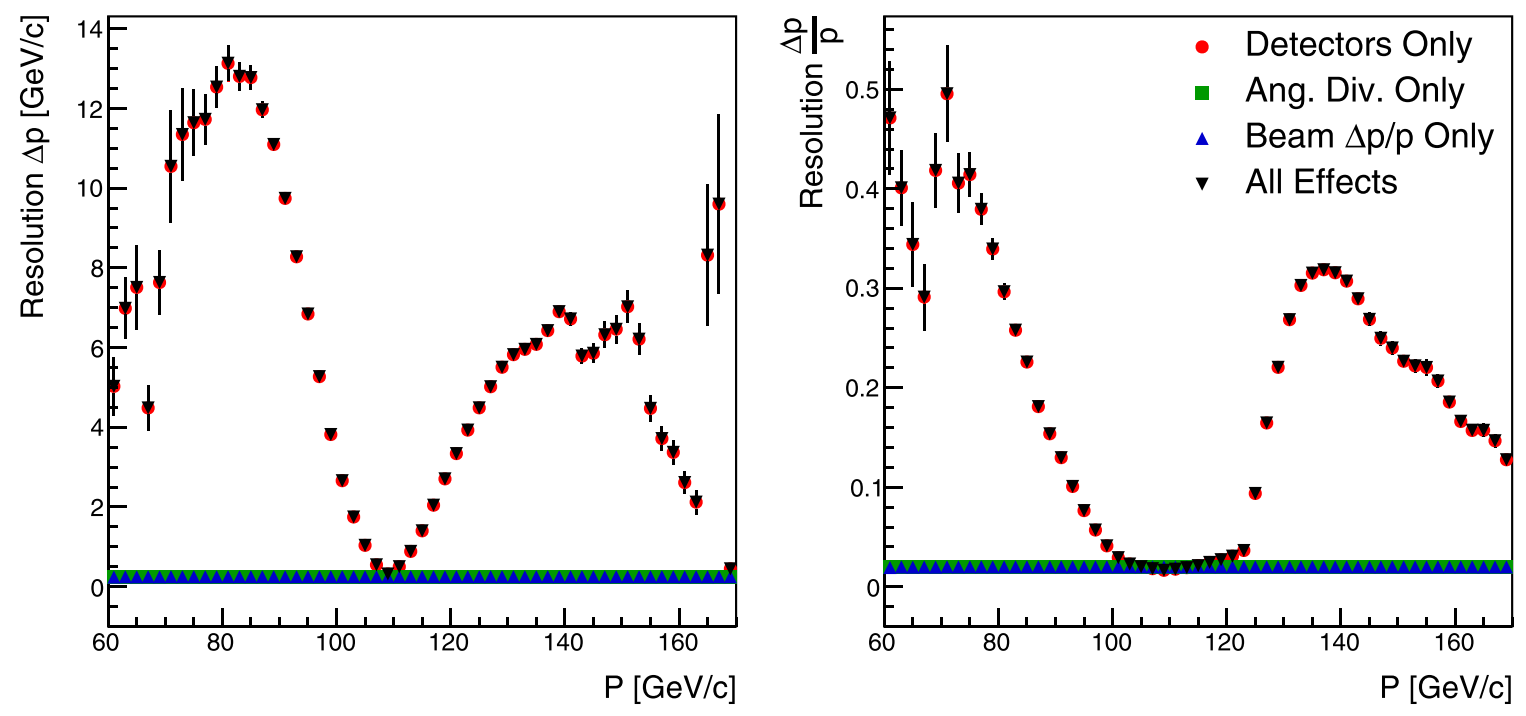

FIG. 15. Absolute (left) and relative (right) total 3-momentum resolution for spectator protons. The plots show the contributions of the various effects and the total resolution. 

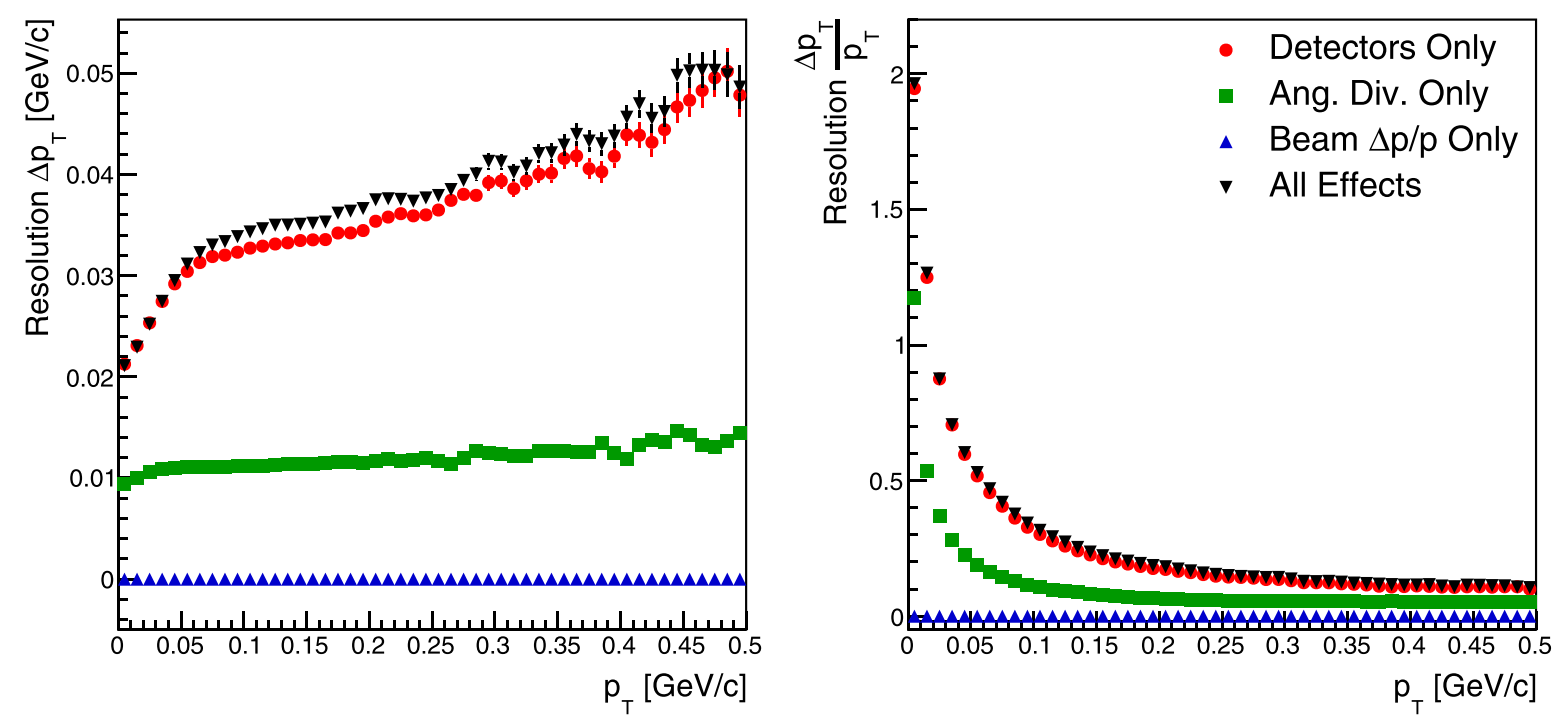

FIG. 16. Absolute (left) and relative (right) transverse momentum resolution for spectator neutrons. The plots show the contributions of the various effects and the total resolution.

Figures 14 and 15 summarize the resolution for spectator protons. Figure 14 shows the proton transverse momentum resolution. One observes that at lower values of $p_{T}$ the angular divergence is the dominant factor in the reconstruction smearing, but quickly becomes sub-dominant to the detector effects at higher $p_{T}$. At higher values of $p_{T}(>100 \mathrm{MeV})$, most of the additional smearing from the detector comes from the assumption of a linear transport matrix with decoupled $x$ and $y$ momentum components, which is an overly simplistic assumption to make in the case where the protons are severely divergent from the beam rigidity. The matrix is tuned for trajectories of protons with $\approx 1 / 2$ rigidity compared to the beam, allowing the smearing effect to be minimized at that point. This will be corrected in a future analysis as more time allows for a more sophisticated approach to be developed, but does not affect the kinematic region relevant to the pole extrapolation presented in this work.

Figures 16 and 17 summarize the corresponding resolutions for neutron spectators. Figure 16 shows the neutron transverse momentum resolution. One observes that the detector resolution accounts for the majority of the overall reconstruction smearing, with the angular divergence contributing to increased reconstruction smearing especially at lower values of $p_{T}$. The beam-momentum uncertainty contributes a negligible amount to the overall reconstruction smearing. Figure 17 shows the neutron total (longitudinal) momentum resolution. Since the angular divergence only acts on the transverse momentum components, the 3-momentum resolution is dominated by the resolution of the detector. The overall momentum smearing in the case of the neutrons is
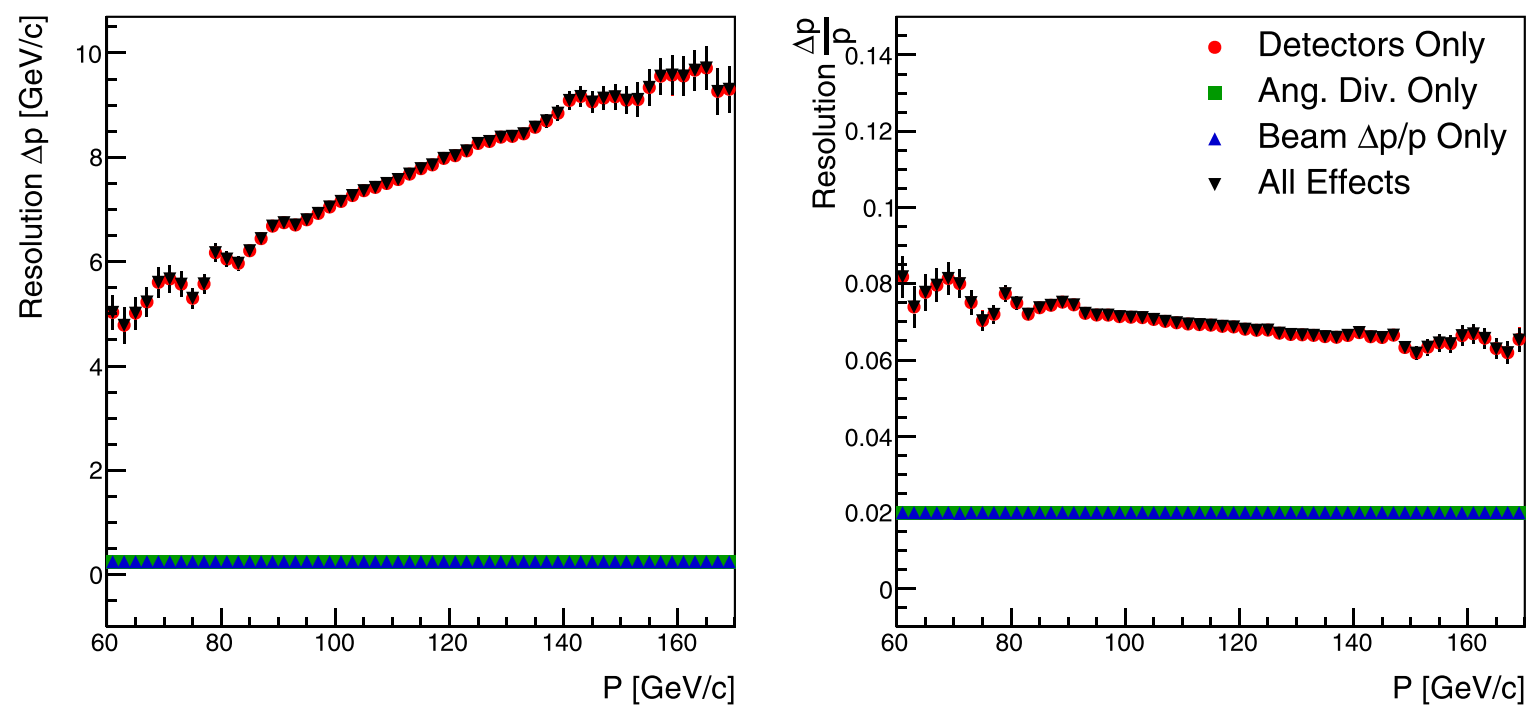

FIG. 17. Absolute (left) and relative (right) total three-momentum resolution for spectator neutrons. The plots show the contributions of the various effects and the total resolution. 

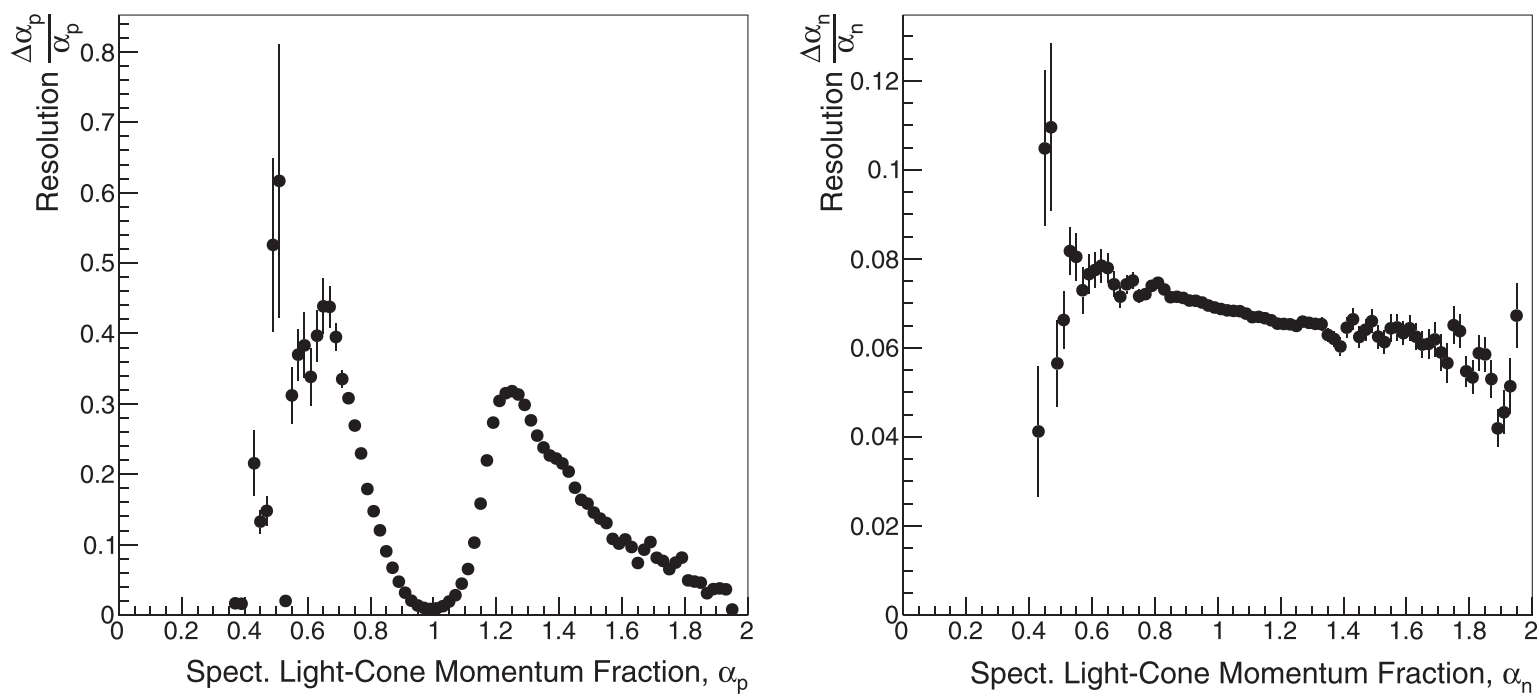

FIG. 18. Resolution in the light-front momentum fraction, $\alpha$, for protons (left) and neutron (right).

worse than for the protons due to the energy resolution under consideration for the zero-degree calorimeter for the EIC, as discussed in Sec. III C. As seen in the case of the proton, the beam-momentum uncertainty plays a negligible role in the overall reconstruction smearing for neutrons.

Figure 18 shows the longitudinal momentum resolution for proton and neutron spectators directly in the physics variables $\alpha_{p}$ and $\alpha_{n}$; see Eqs. (59). One observes that, in the area of interest to the present physics study, $\alpha \approx 1$, the resolution for protons is $<1 \%$, while for neutrons it is closer to $\approx 7 \%$. This difference is essential in assessing the tagged cross section measurements and the different approaches to pole removal in the present study (see Sec. IIF and Fig. 6). We note that in the physics study the simulated resolutions in Fig. 18 were not applied directly, but were used to construct a smearing function that was applied to the data.
[1] J. J. Ethier and E. R. Nocera, Parton distributions in nucleons and nuclei, Annu. Rev. Nucl. Part. Sci. 70, 43 (2020).

[2] J. Gao, L. Harland-Lang, and J. Rojo, The structure of the proton in the LHC precision era, Phys. Rep. 742, 1 (2018).

[3] C. A. Aidala, S. D. Bass, D. Hasch, and G. K. Mallot, The spin structure of the nucleon, Rev. Mod. Phys. 85, 655 (2013).

[4] K. Goeke, M. V. Polyakov, and M. Vanderhaeghen, Hard exclusive reactions and the structure of hadrons, Prog. Part. Nucl. Phys. 47, 401 (2001).

[5] M. Diehl, Generalized parton distributions, Phys. Rep. 388, 41 (2003).

[6] A. V. Belitsky and A. V. Radyushkin, Unraveling hadron structure with generalized parton distributions, Phys. Rep. 418, 1 (2005).

[7] M. Guidal, H. Moutarde, and M. Vanderhaeghen, Generalized parton distributions in the valence region from deeply virtual Compton scattering, Rep. Prog. Phys. 76, 066202 (2013).

[8] A. Bacchetta, M. Diehl, K. Goeke, A. Metz, P. J. Mulders, and M. Schlegel, Semi-inclusive deep inelastic scattering at small transverse momentum, J. High Energy Phys. 02 (2007) 093.

[9] A. Signori, A. Bacchetta, M. Radici, and G. Schnell, Investigations into the flavor dependence of partonic transverse momentum, J. High Energy Phys. 11 (2013) 194.

[10] V. Barone, F. Bradamante, and A. Martin, Transverse-spin and transverse-momentum effects in high-energy processes, Prog. Part. Nucl. Phys. 65, 267 (2010).

[11] L. L. Frankfurt and M. I. Strikman, Hard nuclear processes and microscopic nuclear structure, Phys. Rep. 160, 235 (1988).
[12] D. F. Geesaman, K. Saito, and A. W. Thomas, The nuclear EMC effect, Annu. Rev. Nucl. Part. Sci. 45, 337 (1995).

[13] S. Malace, D. Gaskell, D. W. Higinbotham, and I. Cloet, The challenge of the EMC effect: Existing data and future directions, Int. J. Mod. Phys. E 23, 1430013 (2014).

[14] O. Hen, G. A. Miller, E. Piasetzky, and L. B. Weinstein, Nucleon-nucleon correlations, short-lived excitations, and the quarks within, Rev. Mod. Phys. 89, 045002 (2017).

[15] S. Fucini, M. Rinaldi, and S. Scopetta, Generalized parton distributions of light nuclei, Few Body Syst. 62, 3 (2021).

[16] L. Frankfurt, V. Guzey, and M. Strikman, Leading twist nuclear shadowing phenomena in hard processes with nuclei, Phys. Rep. 512, 255 (2012).

[17] B. Z. Kopeliovich, J. G. Morfin, and I. Schmidt, Nuclear shadowing in electro-weak interactions, Prog. Part. Nucl. Phys. 68, 314 (2013).

[18] J. Adam et al., Electron ion collider conceptual design report (2021), https://www.bnl.gov/ec/files/EIC_CDR_Final.pdf.

[19] R. Abdul Khalek et al., Science requirements and detector concepts for the electron-ion collider: EIC yellow report, arXiv:2103.05419.

[20] L. B. Weinstein, E. Piasetzky, D. W. Higinbotham, J. Gomez, O. Hen, and R. Shneor, Short Range Correlations and the EMC Effect, Phys. Rev. Lett. 106, 052301 (2011).

[21] L. L. Frankfurt and M. I. Strikman, High-energy phenomena, short range nuclear structure and QCD, Phys. Rep. 76, 215 (1981).

[22] M. Sargsian and M. Strikman, Model independent method for determination of the DIS structure of free neutron, Phys. Lett. B 639, 223 (2006). 
[23] M. Strikman and C. Weiss, Electron-deuteron deep-inelastic scattering with spectator nucleon tagging and final-state interactions at intermediate $x$, Phys. Rev. C 97, 035209 (2018).

[24] W. Cosyn and C. Weiss, Polarized electron-deuteron deepinelastic scattering with spectator nucleon tagging, Phys. Rev. C 102, 065204 (2020).

[25] L. L. Frankfurt and M. I. Strikman, High momentum transfer processes with polarized deuterons, Nucl. Phys. A 405, 557 (1983).

[26] W. Cosyn and C. Weiss, Neutron spin structure from polarized deuteron DIS with proton tagging, Phys. Lett. B 799, 135035 (2019).

[27] L. Frankfurt, V. Guzey, and M. Strikman, Nuclear Shadowing and Extraction of $F_{2}^{p}-F_{2}^{n}$ at Small $x$ from Deuteron Collider Data, Phys. Rev. Lett. 91, 202001 (2003).

[28] L. Frankfurt, V. Guzey, and M. Strikman, Nuclear shadowing in inclusive and tagged deuteron structure functions and extraction of $F_{2}^{p}-F_{2}^{n}$ at small $x$ from electron-deuteron collider data, Mod. Phys. Lett. A 21, 23 (2006).

[29] N. Baillie et al. (CLAS Collaboration), Measurement of the Neutron $F_{2}$ Structure Function via Spectator Tagging with CLAS, Phys. Rev. Lett. 108, 142001 (2012); 108, 199902(E) (2012).

[30] S. Tkachenko et al. (CLAS Collaboration), Measurement of the structure function of the nearly free neutron using spectator tagging in inelastic ${ }^{2} \mathrm{H}\left(e, e^{\prime} p\right) X$ scattering with CLAS, Phys. Rev. C 89, 045206 (2014); 90, 059901(E) (2014).

[31] S. Bueltmann et al., The Structure of the Free Neutron at Large $x$-Bjorken, Report JLAB-PR12-06-113 (2006), https:// www.jlab.org/exp_prog/proposals/10/PR12-06-113-pac36.pdf.

[32] W. Armstrong et al., Tagged EMC measurements on light nuclei, arXiv: 1708.00891

[33] A. V. Klimenko et al. (CLAS Collaboration), Electron scattering from high-momentum neutrons in deuterium, Phys. Rev. C 73, 035212 (2006).

[34] O. Hen et al., In Medium Proton Structure Functions, SRC, and the EMC effect, Jefferson Lab Experiment E1211-003A (2015), https://www.jlab.org/exp_prog/proposals/15/ E12-11-003A.pdf

[35] O. Hen, L. Weinstein, S. Gilad, and S. Wood, In medium nucleon structure functions, SRC, and the EMC effect, arXiv:1409.1717.

[36] C. Weiss et al., Jefferson Lab 2014-2015 Laboratorydirected Research and Development Project, https://www.jlab. org/theory/tag/.

[37] W. Cosyn, V. Guzey, M. Sargsian, M. Strikman, and C. Weiss, Electron-deuteron DIS with spectator tagging at EIC: Development of theoretical framework, EPJ Web Conf. 112, 01022 (2016).

[38] E. Aschenauer, M. D. Baker, W. Chang, J. H. Lee, Z. Tu, and L. Zheng, BeAGLE: A Tool to Refine Detector Requirements for eA Collisions EIC R\&D Project eRD17: Progress Report (January-June 2019) and Proposal (2019), https://wiki.bnl.gov/ eic/index.php/BeAGLE.

[39] A. Jentsch, M. Strikman, Z. Tu, and C. Weiss (unpublished).

[40] F. D. Aaron et al. (H1 Collaboration), Measurement of the inclusive $e p$ scattering cross section at low $Q^{2}$ and $x$ at HERA, Eur. Phys. J. C 63, 625 (2009).

[41] W. Cosyn and C. Weiss (unpublished).
[42] M. Arneodo, Nuclear effects in structure functions, Phys. Rep. 240, 301 (1994).

[43] T. Sjöstrand, S. Mrenna, and P. Skands, PYTHIA 6.4 physics and manual, J. High Energy Phys. 05 (2006) 026.

[44] S. Roesler, R. Engel, and J. Ranft, The Monte Carlo event generator DPMJET-III, in Advanced Monte Carlo for Radiation physics, Particle Transport Simulation and Applications, Proceedings of the Monte Carlo 2000 Conference, Lisbon, Portugal, October 23-26, 2000 (Springer, Berlin, 2000), pp. 1033-1038.

[45] T. Böhlen, F. Cerutti, M. Chin, A. Fassò, A. Ferrari, P. Ortega, A. Mairani, P. Sala, G. Smirnov, and V. Vlachoudis, The FLUKA code: Developments and challenges for high energy and medical applications, Nucl. Data Sheets 120, 211 (2014).

[46] A. Ferrari, P. R. Sala, A. Fasso, and J. Ranft, FLUKA: A multiparticle transport code, program version 2005 (unpublished).

[47] Z. Tu, A. Jentsch, M. Baker, L. Zheng, J.-H. Lee, R. Venugopalan, O. Hen, D. Higinbotham, E.-C. Aschenauer, and T. Ullrich, Probing short-range correlations in the deuteron via incoherent diffractive $\mathrm{J} / \psi$ production with spectator tagging at the EIC, Phys. Lett. B 811, 135877 (2020).

[48] C. Ciofi degli Atti and S. Simula, Realistic model of the nucleon spectral function in few and many nucleon systems, Phys. Rev. C 53, 1689 (1996).

[49] A. Kiselev and A. Jentsch, EicRoot: A light-weight GEANT detector simulation software suite based on the FairRoot framework (2020), https://github.com/eic/EicRoot.

[50] S. Agostinelli et al. (GEANT4 Collaboration), GEANT4-a simulation toolkit, Nucl. Instrum. Meth. A 506, 250 (2003).

[51] L. L. Frankfurt and M. I. Strikman, Point-like configurations in hadrons and nuclei and deep inelastic reactions with leptons: EMC and EMC like effects, Nucl. Phys. B 250, 143 (1985).

[52] C. Ciofi degli Atti, L. L. Frankfurt, L. P. Kaptari, and M. I. Strikman, On the dependence of the wave function of a bound nucleon on its momentum and the EMC effect, Phys. Rev. C 76, 055206 (2007).

[53] G. Wolf, Review of high energy diffraction in real and virtual photon proton scattering at HERA, Rep. Prog. Phys. 73, 116202 (2010).

[54] N. Armesto, P. R. Newman, W. Słomiński, and A. M. Staśto, Inclusive diffraction in future electron-proton and electron-ion colliders, Phys. Rev. D 100, 074022 (2019).

[55] V. Guzey, M. Strikman, and C. Weiss (unpublished).

[56] V. Guzey, E. Kryshen, M. Strikman, and M. Zhalov, Evidence for nuclear gluon shadowing from the ALICE measurements of $\mathrm{PbPb}$ ultraperipheral exclusive $J / \psi$ production, Phys. Lett. B 726, 290 (2013).

[57] V. Guzey and M. Zhalov, Exclusive $J / \psi$ production in ultraperipheral collisions at the LHC: Constraints on the gluon distributions in the proton and nuclei, J. High Energy Phys. 10 (2013) 207.

[58] V. Guzey, E. Kryshen, M. Strikman, and M. Zhalov, Nuclear suppression from coherent $J / \psi$ photoproduction at the Large Hadron Collider, Phys. Lett. B 816, 136202 (2021).

[59] D. Boer and P. J. Mulders, Time reversal odd distribution functions in leptoproduction, Phys. Rev. D 57, 5780 (1998).

[60] J. Adam and others, Accelerator and beam conditions critical for physics and detector simulations for the Electron-Ion Collider (2021), http://www.eicug.org/web/documents/public. 
[61] A. Metz and A. Vossen, Parton fragmentation functions, Prog. Part. Nucl. Phys. 91, 136 (2016).

[62] W. Cosyn, V. Guzey, D. W. Higinbotham, C. Hyde, S. Kuhn, P. Nadel-Turonski, K. Park, M. Sargsian, M. Strikman, and C. Weiss, Neutron spin structure with polarized deuterons and spectator proton tagging at EIC, J. Phys.: Conf. Ser. 543, 012007 (2014).

[63] I. Friscic et al., Neutron spin structure from $e-{ }^{3} \mathrm{He}$ scattering with double spectator tagging at the electron-ion collider, Phys. Lett. B 823, 136726 (2021).

[64] T. E. O. Ericson and W. Weise, Pions and Nuclei (Clarendon, Oxford, 1988).

[65] R. B. Wiringa, V. G. J. Stoks, and R. Schiavilla, Accurate nucleon-nucleon potential with charge-independence breaking, Phys. Rev. C 51, 38 (1995).

[66] M. Piarulli, L. Girlanda, R. Schiavilla, R. N. Pérez, J. E. Amaro, and E. R. Arriola, Minimally nonlocal nucleon-nucleon potentials with chiral two-pion exchange including $\Delta$ resonances, Phys. Rev. C 91, 024003 (2015).

[67] C. W. Wong, Deuteron radius and nuclear forces in free space, Int. J. Mod. Phys. E 3, 821 (1994).

[68] W. Chang, E.-C. Aschenauer, M. D. Baker, A. Jentsch, J.-H. Lee, Z. Tu, Z. Yin, and L. Zheng, Investigation of the background in coherent $J / \psi$ production at the EIC, arXiv:2108.01694. 